\title{
Pole dynamics and oscillations for the complex Burgers equation in the small-dispersion limit
}

\author{
D Senouf $\dagger$, R Caflisch $\ddagger$ and N Ercolani§ \\ $\dagger$ Lehman Brothers, One Broadgate, London EC2M 7HA, UK \\ $\ddagger$ Department of Mathematics, University of California, Los Angeles, CA 90095, USA \\ $\S$ Department of Mathematics, University of Arizona, Tucson, AZ 85721, USA
}

Received 29 September 1995

Recommended by J-P Eckmann

\begin{abstract}
A meromorphic solution to the Burgers equation with complex viscosity is analysed. The equation is linearized via the Cole-Hopf transform which allows for a careful study of the behaviour of the singularities of the solution. The asymptotic behaviour of the solution as the dispersion coefficient tends to zero is derived. For small dispersion, the time evolution of the poles is found by numerically solving a truncated infinite-dimensional Calogero-type dynamical system. The initial data are provided by high-order asymptotic approximations of the poles at the critical time $t_{s}$ for the dispersionless solution via the method of steepest descents. The solution is reconstructed using the pole expansion and the location of the poles. The oscillations observed via the singularities are compared to those obtained by a classical stationary phase analysis of the solution as the dispersion parameter $\epsilon \rightarrow 0^{+}$. A uniform asymptotic expansion as $\epsilon \rightarrow 0^{+}$ of the dispersive solution is derived in terms of the Pearcey integral in a neighbourhood of the caustic. A continuum limit of the pole expansion and the Calogero system is obtained, yielding a new integral representation of the solution to the inviscid Burgers equation.
\end{abstract}

AMS classification scheme numbers: 35A20, 35A40, 35B40, 35Q53, 41A60

\section{Introduction}

Many nonlinear dispersive systems exhibit rapid oscillations in their spatial-temporal dependence in the regime of small dispersion. Examples include partial differential equations (PDEs) such as the Korteweg-de Vries (KdV) equation, the nonlinear Schrödinger equation $[16,17,24]$, and finite-difference equations such as the Lax-Wendroff method (see also [25]). Although a fascinating mathematical phenomenon, these oscillations are generally quite difficult to describe and control and are an obstacle to the efficiency of numerical and analytical methods. A complete analysis of oscillations would include a slowly varying description of their shape, amplitude, wavelength and phase. However, these features have been successfully analysed only for a few completely integrable systems such as the KdV equation.

The Burgers equation with an imaginary 'viscosity' coefficient $v=\mathrm{i} \epsilon$, given by

$$
\frac{\partial \psi}{\partial t}+\psi \frac{\partial \psi}{\partial x}=i \epsilon \frac{\partial^{2} \psi}{\partial x^{2}}, \quad \epsilon \geqslant 0
$$

was first described by Dobrokhotov et al in [15]. It is perhaps the simplest example of a nonlinear dispersive equation but has received surprisingly little attention. This equation has the same linear part $\left(\psi_{t}-\mathrm{i} \epsilon \psi_{x x}\right)$ as the Schrödinger equation, and as such can be 
referred to as the Schrödinger equation with convective nonlinearity. We do not know of any applications in which this equation arises, and it does not seem to have a Hamiltonian structure. Moreover, the system is nonlinearly ill posed at least for certain complex values of $v$, since singularities can occur in finite time. Nevertheless, we believe that this equation is an interesting mathematical prototype for dispersive (imaginary $v$ ) or mixed dissipativedispersive systems (complex $v$ ). In particular, this work demonstrates the coexistence of concentration and oscillations in the dispersive dynamics. Such phenomena were seen in numerical simulations of the ID focusing nonlinear Schrödinger equation [22] but the validity of those numerics could not be established. For an introductory discussion on dispersive phenomena, see [33].

In this paper, we present a numerical and analytic study of solutions to (1.1) for complex values of $v$. The solution to equation (1.1) can be solved using the Cole-Hopf nonlinear transform which yields an integral representation involving the heat kernel. For small $|v|=\epsilon$, the resulting formula for $\psi_{\nu}$ can be approximated using the stationary phase method. A new method used to compute the solution is found through pole dynamics. This method is based on obtaining the time-dependent locations of the complex poles of the function $\psi_{v}$ by solving an infinite system of coupled ordinary differential equations (ODEs). The solution $\psi_{v}$ is then found by computing its Mittag-Leffler expansion which involves the position of the poles. One can also compute $\psi_{v}$ directly through a finite-difference method, at least for times before a pole hits the real axis. Finally, in the zero-dispersion (or zero-viscosity limit) $v \rightarrow 0$, the poles coalesce onto a branch cut, and the zero-dispersion solution is described by branch-cut dynamics. This method may be of general interest as a new (to the best of our knowledge) method for solving the inviscid Burgers equation.

These methods will be formulated in general, but they will be numerically evaluated for a special choice of initial data, namely the cubic polynomial

$$
\psi(x, 0)=4 x^{3}-x / t_{*}
$$

which is chosen for its generic features for the inviscid equation $[3,18,29]$. In these initial data, $t_{*}$ is positive and corresponds to the time of first singularity formation for the inviscid problem. The cube root singularity found at the origin at $t=t_{*}$ is known to be a generic singularity for the inviscid Burgers equation. It is due to the coalescence of two conjugate branch points of order two in the complex plane $[3,4,8,18]$. Moreover both cases $v=0$ and $v \neq 0$ can be completely analysed and, in the case $v \neq 0$, there is an instantaneous generation at $t>0$ of a countable set of complex spatial simple poles. For these initial data, the small-dispersion $\left(\epsilon \rightarrow 0^{+}\right)$stationary phase approximation of the solution and its zeros can be evaluated rather explicitly, at least for $t=t_{*}$.

There are three main points to this work. First, in the purely dispersive case in which $v$ is imaginary and small, the solution $\psi_{\nu}$ of (1.1) develops rapid oscillations. Second, these oscillations are caused by the presence of complex poles in $\psi$ which have moved close to the real axis. This result, which is clearly demonstrated below through comparison of the pole dynamics with the solution on the real axis, is important in providing a tangible cause for the formation of the oscillations. Third, the branch-cut dynamics provide a slowly varying but incomplete description of the pole locations. Although we have not yet succeeded in deriving a slowly varying description of the oscillations themselves, we believe that the branch-cut dynamics represent a promising start.

In order to investigate the positions of the poles, we derive a Calogero-type infinitedimensional dynamical system by replacing the pole expansion of the solution into the PDE (see $[10,11]$ for the origin of such a method). We then solve numerically a truncated version of this system, where the initial data are generated by asymptotic and numerical 
approximations of the poles at the inviscid pre-shock time $t_{*}$.

The numerical resolution of the stationary solution of a Calogero dynamical system has previously been used [31] to obtain the stationary positions of the poles of the solution to a flame front equation. In the case $v>0$, the poles are fixed to the imaginary axis and move towards the origin until $t \approx t_{*}$, after which they turn around and move away as $t$ increases $[29$, I]. When dispersion is added, i.e. $\theta=\arg v \neq 0$, the poles are no longer confined to the imaginary axis and evolve in the complex plane describing intricate motions. When $v=\mathrm{i} \epsilon$ $(\epsilon>0)$ is a purely dispersive coefficient, the poles spiral around the real axis. The proximity of the poles to the real axis generates oscillations which are observed by reconstructing the solution numerically via the pole expansion and the pole dynamics. Another approach for tracking complex singularities based on spectral methods can be found in [30].

\section{Integral representation, pole expansion and pole dynamics for $\nu>0$}

We recall some results that have been derived in [29]. In this case we let $\psi_{v}=u_{v}$ to be consistent with the familiar notation that is used in the classical Burgers equation (see $[6,7])$. For $v>0$, the Cole-Hopf transform $u_{v}=-2 v \partial_{x} \log \left(\phi_{v}\right)$ linearizes equation (3.1) into the diffusion equation for $\phi_{v}$ (see $\left.[12,21]\right)$. Thus the solution is given by

$$
\begin{aligned}
& u_{v}(x, t)=\frac{x}{t}-2 v \partial_{x} \log \left(E_{v}(x, t)\right), \\
& E_{v}(x, t)=\int_{-\infty}^{\infty} \exp \left\{\frac{w(z, x)}{2 v}\right\} \mathrm{d} z,
\end{aligned}
$$

where $w(z, x)$ is the phase function defined by

$$
w(z, x)=\int_{0}^{z}\left(\frac{x}{t}-\frac{\eta}{t}-u_{0}(\eta)\right) \mathrm{d} \eta=\frac{x}{t} z+\alpha z^{2}-z^{4},
$$

and $\alpha=\left(t-t_{*}\right) /\left(2 t t_{*}\right) \in \mathbb{R}$. The function $E_{v}(x, t)$ has the following properties for fixed $t, v>0$ :

(i) It is an even entire function of $x$.

(ii) Its order $\lambda=4 / 3$.

(iii) Its genus $h=1$.

(iv) It has infinitely many conjugate and opposite zeros on the imaginary axis.

(v) The order of convergence of the zeros is the order $\lambda=4 / 3$, i.e. $\forall \varepsilon>0$, $\sum_{n} 1 /\left|a_{n}\right|^{\lambda+\varepsilon}<+\infty$.

The fact that the zeros are imaginary has been proved by Pólya [27]. Combining these properties, $E_{v}(x, t)$ has an infinite product representation in terms of its zeros which we denote by $x= \pm a_{n}= \pm \mathrm{i} \beta_{n}$. Since these zeros satisfy the convergence criteria $\sum_{n} \beta_{n}^{-2}<+\infty$ and $\sum_{n} \beta_{n}^{-1}=+\infty$, we have

$$
\begin{aligned}
& E_{v}(x, t)=\mathcal{C}_{v}(t) \prod_{n=1}^{\infty}\left(1+\frac{x^{2}}{\beta_{n}^{2}(t, v)}\right) \\
& \mathcal{C}_{v}(t)=E_{v}(0, t)=\int_{-\infty}^{+\infty} \mathrm{e}^{\frac{1}{2 v}\left(\alpha y^{2}-y^{4}\right)} \mathrm{d} y=\frac{\sqrt{\alpha}}{2} \mathrm{e}^{\frac{\alpha^{2}}{16 v}} K_{1 / 4}\left(\frac{\alpha^{2}}{16 v}\right),
\end{aligned}
$$

where $\mathcal{C}_{v}\left(t_{*}\right)=v^{1 / 4} 2^{-3 / 4} \Gamma(1 / 4), K_{q}(z)$ is the modified Bessel function of the second kind (see [1] for the definition of $K_{q}(z)$ ), and $K_{1 / 4}(z)=\mathrm{O}\left(z^{-1 / 4}\right)$ as $z \rightarrow 0$. After logarithmic differentiation of $E_{v}$, using (2.1a) and (2.2a), the singular part of the solution being the ratio of two entire functions with zeros is meromorphic. Thus we find an infinite pole expansion 
of Mittag-Leffler type for the solution which converges uniformly on compact sets for $x$ away from the poles $x= \pm \mathrm{i} \beta_{n}$ :

$$
u_{v}(x, t)=\frac{x}{t}-\frac{U_{v}(x, t)}{t}=\frac{x}{t}-\sum_{n=1}^{\infty} \frac{4 v x}{x^{2}+\beta_{n}^{2}(t, v)},
$$

where $U_{v}(x, t)$ is the spatially singular part of the viscous solution defined by

$$
U_{v}(x, t)=x-t u_{v}(x, t)=t \sum_{n=1}^{\infty} \frac{4 v x}{x^{2}+\beta_{n}^{2}(t, v)} .
$$

Note that $u_{v}$ can be expressed in a more symmetric way as

$$
u_{v}(x, t)=\frac{x}{t}-2 v \sum_{\substack{n=-\infty \\ n \neq 0}}^{\infty} \frac{1}{x-\mathrm{i} \beta_{n}(t, v)},
$$

where we use the convention that $\beta_{-n}=-\beta_{n}$. Let

$$
\forall n \in \mathbb{N}^{*}=\mathbb{N} \backslash\{0\}, \quad \dot{\beta}_{n}=\frac{\mathrm{d} \beta_{n}}{\mathrm{~d} t},
$$

then we replace the full Mittag-Leffler (pole) expansion found in (2.3) in the PDE $u_{t}+u u_{x}=v u_{x x}$. Using partial fraction expansions, we find (see [29] for more details)

$$
\dot{\beta}_{n}=\frac{\beta_{n}}{t}+\frac{v}{\beta_{n}}-2 v \sum_{\substack{l=1 \\ l \neq n}}^{\infty} \frac{2 \beta_{n}}{\beta_{l}^{2}-\beta_{n}^{2}} .
$$

Similarly to (2.5), there is a more symmetric formulation to the dynamical system (2.7) given by

$$
\dot{\beta}_{n}=\frac{\beta_{n}}{t}-2 v \sum_{\substack{l=-\infty \\ l \neq n, 0}}^{\infty} \frac{1}{\beta_{l}-\beta_{n}} .
$$

Note that the pole expansion (2.5) and the dynamical system (2.8) represent a general solution to the Burgers equation which is independent of the initial data.

Multiplying (2.7) by $\beta_{n}$ and introducing the variable

$$
\gamma_{n}(t, v)=\frac{\beta_{n}^{2}(t, v)}{v}
$$

we have $\sum_{n} \gamma_{n}^{-1}<+\infty$, and system (2.7) becomes independent of $v$ :

$$
\forall n \in \mathbb{N}^{*}, \quad \frac{\dot{\gamma}_{n}}{2}=\frac{\gamma_{n}}{t}+1-4 \gamma_{n} \sum_{\substack{l=1 \\ l \neq n}}^{\infty} \frac{1}{\gamma_{l}-\gamma_{n}} .
$$

\section{Integral representation, pole expansion and pole dynamics for $\nu \in \mathbb{C}^{+}$}

In the analysis that follows, we take advantage of the complete integrability of the Burgers model of a one-dimensional fluid and allow for the viscosity coefficient $v$ to take complex values of the form $v=\epsilon \mathrm{e}^{\mathrm{i} \theta}, \epsilon>0$ and $|\theta| \leqslant \pi / 2$. Then $\psi=\psi_{v}(x, t)$ satisfies

$$
\frac{\partial \psi}{\partial t}+\psi \frac{\partial \psi}{\partial x}=v \frac{\partial^{2} \psi}{\partial x^{2}}, \quad x \in \mathbb{R}, t>0, v \in \mathbb{C}^{+},
$$

where

$$
\mathbb{C}^{+} \equiv\{v \in \mathbb{C} \text { s.t. }|\nu|>0 \text { and }|\arg v| \leqslant \pi / 2\}=\{v \mid \Re v \geqslant 0, v \neq 0\} .
$$


We can express this complex PDE as a system of two real coupled PDEs. Let $\psi=\psi_{R}+\mathrm{i} \psi_{I}$ where $\psi_{R}=\Re \psi$ and $\psi_{I}=\Im \psi$; then (3.1) becomes

$\partial_{t}\left(\begin{array}{l}\psi_{R} \\ \psi_{I}\end{array}\right)+\left(\begin{array}{cc}\psi_{R} & -\psi_{I} \\ \psi_{I} & \psi_{R}\end{array}\right) \partial_{x}\left(\begin{array}{l}\psi_{R} \\ \psi_{I}\end{array}\right)=\epsilon\left(\begin{array}{cc}\cos \theta & -\sin \theta \\ \sin \theta & \cos \theta\end{array}\right) \partial_{x x}\left(\begin{array}{l}\psi_{R} \\ \psi_{I}\end{array}\right)$.

When this coefficient is purely imaginary, $v=\mathrm{i} \epsilon, \epsilon>0$, (3.3) can be thought of as the nonlinear Schrödinger equation with convective nonlinearity:

$$
\frac{\partial \psi}{\partial t}+\psi \frac{\partial \psi}{\partial x}=\mathrm{i} \epsilon \frac{\partial^{2} \psi}{\partial x^{2}}, \quad x \in \mathbb{C}, t>0, \epsilon \geqslant 0 .
$$

From the integral definition of $E_{v}(x, t)$ in $(2.1 b)$, one can extend the $v$ domain of validity to complex values of $v$. Let $v=\epsilon \mathrm{e}^{\mathrm{i} \theta} \in \mathbb{C}^{+}$then

$$
E_{\nu}(x, t)=E_{\epsilon \mathrm{e}^{\mathrm{i} \theta}}(x, t)=\int_{-\infty}^{\infty} \exp \left\{\frac{\mathrm{e}^{-\mathrm{i} \theta}}{2 \epsilon}\left(\frac{x}{t} y+\alpha y^{2}-y^{4}\right)\right\} \mathrm{d} y .
$$

It is straightforward that, in order for the integral (3.5) to remain convergent, we must have $\Re \nu \geqslant 0$, i.e. $|\theta| \leqslant \pi / 2$. This can be verified by using Jordan's lemma and deforming the contour of integration along the ray $\arg y=\theta / 4$ for $0 \leqslant \theta \leqslant \pi / 2$ so that $E_{v}(x, t)$ can be written as

$$
E_{\nu}(x, t)=\mathrm{e}^{\mathrm{i} \theta / 4} \int_{-\infty}^{\infty} \exp \left\{\frac{1}{2 \epsilon}\left(\frac{x}{t} y \mathrm{e}^{-\mathrm{i} 3 \theta / 4}+\alpha \mathrm{e}^{-\mathrm{i} \theta / 2} y^{2}-y^{4}\right)\right\} \mathrm{d} y .
$$

Thus in the range $0 \leqslant \theta \leqslant \pi / 2$, the function $E_{v}(x, t)$ is again an entire function of $x$ of order $\lambda=4 / 3$, and as such it also has infinitely many zeros [2,5]. Noticing the symmetry relation

$$
E_{\bar{v}}(x, t)=\overline{E_{v}(\bar{x}, t)},
$$

we can extend the domain of validity of representation (3.6) to the range $|\theta| \leqslant \pi / 2$. The even parity of $E_{v}(x, t)$ as a function of $x$ is preserved so that

$$
E_{v}(-x, t)=E_{v}(x, t)
$$

The zeros of $E_{v}(x, t)$ therefore come in opposite pairs $x_{n}= \pm a_{n}(t, v)$, with the property that, for each fixed $t>0$ and fixed $v \in \mathbb{C}^{+}$,

$$
\sum_{n} \frac{1}{\left|a_{n}\right|}=+\infty, \quad \sum_{n} \frac{1}{\left|a_{n}\right|^{2}}<+\infty .
$$

The infinite product representation of $E_{v}$ is now

$$
E_{v}(x, t)=\mathcal{C}_{v}(t) \prod_{n=1}^{\infty}\left(1-\frac{x^{2}}{a_{n}^{2}(t, v)}\right),
$$

so that the Mittag-Leffler (pole) expansion becomes

$$
\psi_{v}(x, t)=\frac{x}{t}-\frac{\Psi_{v}(x, t)}{t}=\frac{x}{t}-\sum_{n=1}^{\infty} \frac{4 v x}{x^{2}-a_{n}^{2}(t, v)},
$$

where the spatially singular part of the pole expansion is given by

$$
\Psi_{v}(x, t)=x-t \Psi_{v}(x, t)=t \sum_{n=1}^{\infty} \frac{4 v x}{x^{2}-a_{n}^{2}(t, v)} .
$$

As in (2.5), $\psi_{\nu}$ can be expressed in a more symmetric way as

$$
\psi_{\nu}(x, t)=\frac{x}{t}-2 v \sum_{\substack{n=-\infty \\ n \neq 0}}^{\infty} \frac{1}{x-a_{n}(t, v)} .
$$


Letting $\beta_{n}=-\mathrm{i} a_{n}$ in (2.7), one finds the associated Calogero dynamical system for arbitrary $v \in \mathbb{C}^{+}$. Let

$$
\dot{a}_{n}=\frac{\mathrm{d} a_{n}}{\mathrm{~d} t}, \quad a_{-n}=-a_{n}
$$

then

$$
\dot{a}_{n}=\frac{a_{n}}{t}-\frac{v}{a_{n}}-2 v \sum_{\substack{l=1 \\ l \neq n}}^{\infty} \frac{2 a_{n}}{a_{n}^{2}-a_{l}^{2}}, \quad \forall n \in \mathbb{N}^{*} .
$$

As in (2.8), one can express (3.13) in a more symmetric way as

$$
\dot{a}_{n}=\frac{a_{n}}{t}-2 v \sum_{\substack{l=-\infty \\ l \neq n, 0}}^{\infty} \frac{1}{a_{n}-a_{l}}, \quad \forall n \in \mathbb{N}^{*} .
$$

Note finally that the pole expansion (3.12) and the dynamical system (3.14) represent a general solution to the Burgers equation which is independent of the initial data.

One can further simplify (3.13) by multiplying both sides by $a_{n}$ and introducing the variable

$$
\kappa_{n}=\frac{a_{n}^{2}}{v} .
$$

The corresponding system of ODEs (3.13) becomes free of $v$ so that

$$
\frac{1}{2} \frac{\mathrm{d} \kappa_{n}}{\mathrm{~d} t}=\frac{\dot{\kappa}_{n}}{2}=\frac{\kappa_{n}}{t}-1-4 \kappa_{n} \sum_{\substack{l=1 \\ l \neq n}}^{\infty} \frac{1}{\kappa_{n}-\kappa_{l}}, \quad \forall n \in \mathbb{N}^{*} .
$$

\section{Exact pole locations at $\boldsymbol{t}_{*}$ for $\nu \in \mathbb{C}^{+}$}

At $t=t_{*}$, since $\alpha=0$, we have for $|x|<\infty$

$$
E_{v}\left(x, t_{*}\right)=\mathrm{e}^{\mathrm{i} \theta / 4} \int_{-\infty}^{\infty} \exp \left\{\frac{1}{2 \epsilon}\left(\frac{x}{t_{*}} \mathrm{e}^{-\mathrm{i} 3 \theta / 4} y-y^{4}\right)\right\} \mathrm{d} y .
$$

Thus using Pólya's [27] theorem once more, the zeros of $E_{v}\left(x, t_{*}\right)$ denoted by $\pm a_{n}\left(t_{*}, v\right)$ are located on the ray $\arg x=3 \theta / 4+\pi / 2$, with absolute value $\left|a_{n}\left(t_{*}, \nu\right)\right|=\beta_{n}\left(t_{*}, \epsilon\right)>0$, where $\pm \mathrm{i} \beta_{n}\left(t_{*}, \epsilon\right)$ is the $n$th ordered zero of $E_{\epsilon}\left(x, t_{*}\right)$ on the imaginary axis. For $v=\epsilon \mathrm{e}^{\mathrm{i} \theta} \in \mathbb{C}^{+}$, the zeros of $E_{v}(x, t)$ are thus located at the complex positions

$$
x= \pm a_{n}\left(t_{*}, v=\epsilon \mathrm{e}^{\mathrm{i} \theta}\right)= \pm \mathrm{e}^{\mathrm{i} 3 \theta / 4} \mathrm{i} \beta_{n}\left(t_{*}, \epsilon\right), \quad \forall n \in \mathbb{N}^{*} .
$$

See figure 1 for the positions of the poles at $t=t_{*}$.

Thus, in order to describe the asymptotic behaviour of the zeros of $E_{v}\left(x, t_{*}\right)$, we place ourselves on the ray $\arg x=3 \theta / 4+\pi / 2$, so that at the pre-shock time $t_{*}$, letting $v=\epsilon \mathrm{e}^{\mathrm{i} \theta}$,

$$
\begin{aligned}
E_{\epsilon \mathrm{e}^{\mathrm{i} \theta}}\left(\mathrm{e}^{\mathrm{i} 3 \theta / 4} \mathrm{i} \beta, t_{*}\right) & =\mathrm{e}^{\mathrm{i} \theta / 4} \int_{-\infty}^{\infty} \exp \left\{\frac{1}{2 \epsilon}\left(\frac{\mathrm{i} \beta}{t_{*}} y-y^{4}\right)\right\} \mathrm{d} y \\
& =\mathrm{e}^{\mathrm{i} \theta / 4} E_{\epsilon}\left(\mathrm{i} \beta, t_{*}\right) \\
& =\mathrm{e}^{\mathrm{i} \theta / 4}\left(\frac{\beta}{4 t_{*}}\right)^{1 / 3} F\left(\frac{1}{2 \epsilon}\left(\frac{\beta}{4 t_{*}}\right)^{4 / 3}\right),
\end{aligned}
$$

where we have used the change of variable

$$
y \rightarrow\left(\frac{\beta}{4 t_{*}}\right)^{1 / 3} z,
$$




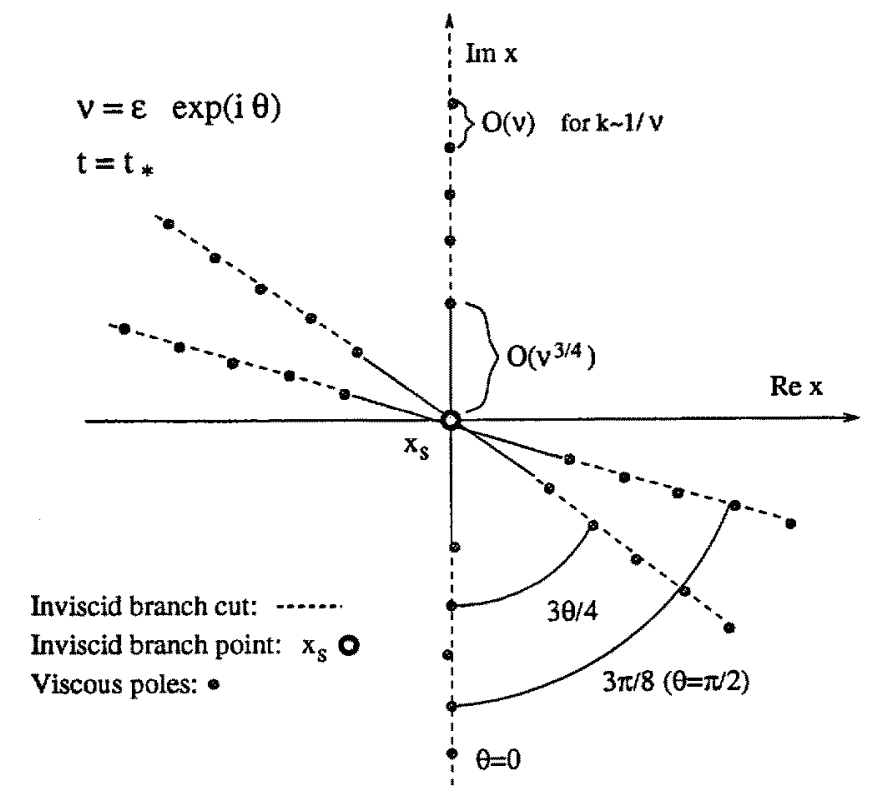

Figure 1. Inviscid branch point, branch cuts and viscous poles at $t=t_{*}$ for $v=|v| \mathrm{e}^{\mathrm{i} \theta}$ $(|\theta| \leqslant \pi / 2)$.

and the function $F(\mu)$ is defined as

$$
F(\mu)=\int_{-\infty}^{\infty} \mathrm{e}^{\mu\left(4 \mathrm{i} z-z^{4}\right)} \mathrm{d} z
$$

Once the zeros $\left\{\mu_{k}\right\}_{k=1}^{\infty}$ of $F(\mu)$ are found, the magnitude $\beta_{k}$ of the zeros $\pm \mathrm{i} \beta_{k}$ of $E_{\epsilon}\left(\mathrm{i} \beta, t_{*}\right)$ are given by the relation

$$
\beta=\beta_{k}\left(t_{*}, \epsilon\right)=4 t_{*}\left(2 \epsilon \mu_{k}\right)^{3 / 4} .
$$

Thus, from (4.2), the zeros of $E_{v}\left(x, t_{*}\right)$ are located at

$$
x= \pm a_{k}\left(t_{*}, v\right)= \pm \mathrm{e}^{\mathrm{i} 3 \theta / 4} \mathrm{i} \beta_{k}\left(t_{*}, \epsilon\right)= \pm \mathrm{e}^{\mathrm{i} 3 \theta / 4} \mathrm{i} 4 t_{*}\left(2 \epsilon \mu_{k}\right)^{3 / 4} .
$$

Using the method of steepest descents described in [13], it is shown in [28] that the $k$ th ordered large zero $\mu_{k}$ of $F(\mu)$ is given as follows.

\section{Property 4.1 Let}

$$
\mu_{k}^{(0)}=\frac{2 \pi}{3 \sqrt{3}}(k-1 / 3), \quad k \geqslant 1
$$

and

$$
G(\mu)=\mu+\frac{7}{432 \mu}\left(1-\frac{1}{6 \mu}\left(1+\frac{7}{72 \mu}\left(1-\frac{5}{12 \mu}\left(1+\frac{53143}{18900 \mu}\right)\right)\right)\right),
$$

then

$$
\mu_{k}=G\left(\mu_{k}^{(0)}\right)+\mathrm{O}\left(\frac{1}{k^{6}}\right) \quad \text { as } k \rightarrow+\infty
$$


5. Asymptotic analysis of $\Psi_{\nu}(x, t)$ for $\nu=i \epsilon$, as $\epsilon \rightarrow 0^{+}, t>t_{*}$

When $v=\mathrm{i} \epsilon, \epsilon>0$, we evaluate the asymptotic behaviour of $E_{v}$ as $\epsilon \rightarrow 0^{+}$using the stationary phase method. We find that all three saddle points are relevant within the caustic $|x|<\left|x_{S}(t)\right|-\delta / 2$, where $\delta>0$ and where $\pm x_{s}(t)$ are the second-order branch points of the inviscid solution [29, I]. For a discussion on such caustics, see [23,26]. When $t>t_{*}$, $x \in\left(-\infty,-x_{s}(t)-\delta / 2\right) \cup\left(x_{s}(t)+\delta / 2, \infty\right), v=\mathrm{i} \epsilon, \epsilon \rightarrow 0^{+}$, the same analysis holds and one recovers the characteristic solution outside the caustic consisting of one relevant saddle point. The transition from within the caustic to outside is not uniform as the asymptotic behaviour at the caustic $x= \pm x_{s}(t)$ is degenerate (two saddle points have coalesced).

\subsection{Asymptotic expansion within the caustic $x \in\left(-x_{s}(t)+\delta / 2, x_{s}(t)-\delta / 2\right), \delta>0$}

The caustic $x=x_{s}(t)$ corresponds to the envelope of the characteristics of the inviscid Burgers solution and is also determined by the system of equations

$$
\begin{aligned}
& 0=w_{z}(z, x)=x / t+2 \alpha z-4 z^{3}, \\
& 0=w_{z z}(z, x)=2 \alpha-12 z^{2},
\end{aligned}
$$

where $w(z, x)$ is the phase function of the integrand in the definition of $E_{v}(x, t)$. This system represents the conditions for the phase function $w$ to have saddle points of multiplicity two, thereby yielding a curve in the $(x, t)$ plane on which two saddles of multiplicity one coalesce into a saddle of multiplicity two. From the second equation in (5.1), we find $z_{\text {caustic }}(t)= \pm \sqrt{\alpha / 6}$ and, from the first,

$$
x=x_{\text {caustic }}=t\left(4 z_{\text {caustic }}(t)^{3}-2 \alpha z_{\text {caustic }}(t)\right)=\mp t\left(\frac{2 \alpha}{3}\right)^{3 / 2}=\mp x_{s}(t),
$$

where $x_{s}(t)=\mathrm{i}\left(3 t_{*}\right)^{-3 / 2}\left(t_{*}-t\right)^{3 / 2} t^{-1 / 2}$ is the second-order branch point of the dispersionless solution described in [29, I]. Here we are only concerned with the dominant behaviour of $E_{\mathrm{i} \epsilon}$, thus we retain only the first term:

$$
E_{\mathrm{i} \epsilon}(x, t)=\sum_{s=0,1,2} \sqrt{\frac{-4 \pi \mathrm{i} \epsilon}{w_{z z}\left(z_{s}, x\right)}} \exp \left(\frac{w\left(z_{s}, x\right)}{2 \mathrm{i} \epsilon}\right)(1+\mathrm{O}(\epsilon)),
$$

as $\epsilon \rightarrow 0^{+}$, with

$$
\begin{aligned}
& w\left(z_{s}(x, t), x\right)=\frac{x}{t} z_{s}+\alpha z_{s}^{2}-z_{s}^{4}=\frac{3}{4} \frac{x}{t} z_{s}+\frac{\alpha}{2} z_{s}^{2}, \\
& w_{z}\left(z_{s}(x, t), x\right)=0, \quad w_{z z}\left(z_{s}(x, t), x\right)=2 \alpha-12 z_{s}^{2} .
\end{aligned}
$$

The values of the saddle points $z_{s}=z_{s}(x, t)$ of (3.6) are determined by the three roots of the first equation in system (5.1), i.e. the first equation of $(5.4 b)$. They are specifically

$$
\begin{aligned}
& z_{0}=\omega \mathcal{A}+\omega^{2} \mathcal{B} \\
& z_{1}=\omega^{2} \mathcal{A}+\omega \mathcal{B} \\
& z_{2}=\mathcal{A}+\mathcal{B}
\end{aligned}
$$

with $w=\mathrm{e}^{2 \pi \mathrm{i} / 3}$ and

$$
\begin{aligned}
& \mathcal{A}(x, t)=(8 t) \sqrt[-1 / 3]{\sqrt[3]{x+\sqrt{x^{2}-x_{s}^{2}}}}, \\
& \mathcal{B}(x, t)=(8 t)^{-1 / 3} \sqrt[3]{x-\sqrt{x^{2}-x_{s}^{2}}} .
\end{aligned}
$$


Note that all three saddle points are real when $x, x_{s} \in \mathbb{R}$ and the discriminant $\Delta=x^{2}-x_{s}^{2}<$ 0 , that is $|x|<\left|x_{s}(t)\right|$, and in this case $\mathcal{A}=\overline{\mathcal{B}}$ [29, appendix B]. Therefore we have $z_{s} \in \mathbb{R}$, $w\left(z_{s}, x\right) \in \mathbb{R}$, and $w_{z z}\left(z_{s}, x\right)=2 \alpha-12 z_{s}^{2} \in \mathbb{R}$. Hence all three terms in the summation signs are oscillatory and equally relevant. Note, however, that the expansion derived for $E_{\mathrm{i} \epsilon}$ is only valid within $|x|<\left|x_{s}\right|$ and, in order to get an expansion uniformly valid across $x= \pm x_{s}$ one needs to derive a uniform expansion as presented in [15]. This analysis is similar in spirit to the one of Jin et al [22 (section 2.2)] and that of [15]. The dominant behaviour of the solution $\psi_{\mathrm{i} \epsilon}(x, t)$ is found from the Cole-Hopf representation, so that within the caustic $|x|<\left|x_{s}\right|-\delta / 2$, following the derivation presented in [29 (I, section 3)], we find that

$$
\begin{aligned}
\Psi_{\mathrm{i} \epsilon}(x, t) & =\frac{\sum_{s=0,1,2} z_{s} \mathrm{e}^{\frac{w(z s, x)}{2 \mathrm{i} \epsilon}} / \sqrt{w_{z z}\left(z_{s}, x\right)}}{\sum_{s=0,1,2} \mathrm{e}^{\frac{w(z s, x)}{2 \mathrm{i} \epsilon}} / \sqrt{w_{z z}\left(z_{s}, x\right)}}+\mathrm{O}(\epsilon) \\
& =\frac{\sum_{s=0,1,2} z_{s} \mathrm{e}^{\frac{w(z, x)}{2 \mathrm{i} \epsilon}-\frac{\mathrm{i}}{2} \arg \left(w_{z z}\left(z_{s}, x\right)\right)}\left|w_{z z}\left(z_{s}, x\right)\right|^{-1 / 2}}{\sum_{s=0,1,2} \mathrm{e}^{\frac{w(z, x)}{2 \mathrm{i} \epsilon}-\frac{\mathrm{i}}{2} \arg \left(w_{z z}\left(z_{s}, x\right)\right)}\left|w_{z z}\left(z_{s}, x\right)\right|^{-1 / 2}}+\mathrm{O}(\epsilon) .
\end{aligned}
$$

Since $w_{z z}\left(z_{s}, x\right) \in \mathbb{R}$, we have that $\arg \left(w_{z z}\left(z_{s}, x\right)\right)=(\pi / 2)\left(1-\operatorname{sgn}\left(w_{z z}\left(z_{s}, x\right)\right)\right)$, and therefore the small-dispersion behaviour of the solution is found from (3.10).

Property 5.1 As $\epsilon \rightarrow 0^{+}$for $x \in\left(-x_{s}(t)+\delta / 2, x_{s}(t)-\delta / 2\right), \delta>0, t>t_{*}$, the spatially singular part of the solution to the Burgers equation is approximated by

$$
\Psi_{\mathrm{i} \epsilon}(x, t)=\frac{\sum_{s=0,1,2} z_{s} \mathrm{e}^{-\frac{\mathrm{i}}{2 \epsilon} w\left(z_{s}, x\right)+\frac{\mathrm{i} \pi}{4} \operatorname{sgn}\left(w_{z z}\left(z_{s}, x\right)\right)}\left|w_{z z}\left(z_{s}, x\right)\right|^{-1 / 2}}{\sum_{s=0,1,2} \mathrm{e}^{-\frac{\mathrm{i}}{2 \epsilon} w\left(z_{s}, x\right)+\frac{\mathrm{i} \pi}{4} \operatorname{sgn}\left(w_{z z}\left(z_{s}, x\right)\right)}\left|w_{z z}\left(z_{s}, x\right)\right|^{-1 / 2}}+\mathrm{O}(\epsilon) .
$$

The asymptotic behaviour of the solution is then found from the relation

$$
\psi_{\mathrm{i} \epsilon}(x, t)=\frac{x}{t}-\frac{\Psi_{\mathrm{i} \epsilon}(x, t)}{t} .
$$

Thus the presence of three competing oscillatory terms in the asymptotic behaviour of $\Psi_{\mathrm{i} \epsilon}$ is reminiscent of the oscillations observed in the solution $\psi_{\mathrm{i} \epsilon}$. Such oscillations are also seen in the pole dynamics in section 7.2.

5.1.1. Long-time asymptotics of the stationary phase solution within the caustic In this section we approximate the stationary phase formula in property 5.1 for small values of $\delta=x / t$ and find the approximate pole positions for large time. First we claim that the stationary phase formula is valid in a complex neighbourhood of $x=0$ independent of $\epsilon=|\nu|$. A full extension of this formula to the complex plane is difficult to determine because of the possibility of Stokes lines [26]. Across a Stokes line a stationary point loses its accessibility, i.e. the ability to deform the integration contour to the steepest descent path through the stationary point is lost. The point $x=0$ does not lie on a Stokes line, however, so that all three stationary points are accessible in a neighbourhood of $x=0$.

Now we can expand the three solutions $y=y_{0}, y_{+}, y_{-}$of $w_{y}=0$, and the corresponding values of $w$ and $w_{y y}$, in powers of $\delta=x / t$ for fixed value of $\alpha=\left(t-t_{*}\right) /\left(2 t t_{*}\right)$ as $\delta \rightarrow 0$ :

$$
\begin{aligned}
& y_{0}=-\frac{\delta}{2 \alpha}+\mathrm{O}\left(\delta^{3}\right) \\
& y_{+}=\sqrt{\frac{\alpha}{2}}+\frac{\delta}{4 \alpha}-\frac{\delta^{2}}{\alpha^{5 / 2}} \frac{3}{32 \sqrt{2}}+\mathrm{O}\left(\delta^{3}\right) \\
& y_{-}=-\sqrt{\frac{\alpha}{2}}+\frac{\delta}{4 \alpha}+\frac{\delta^{2}}{\alpha^{5 / 2}} \frac{3}{32 \sqrt{2}}+\mathrm{O}\left(\delta^{3}\right)
\end{aligned}
$$




$$
\begin{aligned}
& w_{0}=-\frac{\delta^{2}}{4 \alpha}+\mathrm{O}\left(\delta^{3}\right) \\
& w_{+}=\frac{\alpha^{2}}{4}+\delta \sqrt{\frac{\alpha}{2}}+\frac{\delta^{2}}{8 \alpha}+\mathrm{O}\left(\delta^{3}\right) \\
& w_{-}=\frac{\alpha^{2}}{4}-\delta \sqrt{\frac{\alpha}{2}}+\frac{\delta^{2}}{8 \alpha}+\mathrm{O}\left(\delta^{3}\right) \\
& w_{0 y y}=2 \alpha+\mathrm{O}\left(\delta^{2}\right) \\
& w_{+y y}=-4 \alpha-3 \delta \sqrt{\frac{2}{\alpha}}+\mathrm{O}\left(\delta^{2}\right) \\
& w_{-y y}=-4 \alpha+3 \delta \sqrt{\frac{2}{\alpha}}+\mathrm{O}\left(\delta^{2}\right)
\end{aligned}
$$

The stationary phase formula is much more sensitive to the value of $w$ than to the value of $w_{y y}$, and its leading order form is determined by the terms up to $\mathrm{O}(\delta)$ in $w$ and up to $\mathrm{O}(1)$ in $w_{y y}$. In particular the leading order form for the denominator $D$ is (for $v=\mathrm{i} \epsilon$ )

$$
\begin{aligned}
D & =\sum_{s=0,+,-} \sqrt{-\frac{v}{w_{s y y}}} \mathrm{e}^{w_{s} / 2 v} \\
& =\sqrt{\frac{\mathrm{i}}{\epsilon}}\left\{-\mathrm{i}\left|w_{0 y y}\right|^{-1 / 2} \mathrm{e}^{-\mathrm{i} w_{0} / 2 \epsilon}+\left|w_{+y y}\right|^{-1 / 2} \mathrm{e}^{-\mathrm{i} w_{+} / 2 \epsilon}+\left|w_{-y y}\right|^{-1 / 2} \mathrm{e}^{-\mathrm{i} w_{-} / 2 \epsilon}\right\} \\
& =\frac{1}{2} \sqrt{\frac{\mathrm{i} \epsilon}{|\alpha|}}\left\{-\mathrm{i} \sqrt{2}+\mathrm{e}^{-\mathrm{i} w_{+} / 2 \epsilon}+\mathrm{e}^{-\mathrm{i} w_{-} / 2 \epsilon}\right\} \\
& =\frac{1}{2} \sqrt{\frac{\mathrm{i} \epsilon}{|\alpha|}}\left\{-\mathrm{i} \sqrt{2}+2 \cos \left(\frac{\delta}{2 \epsilon} \sqrt{\frac{\alpha}{2}}\right) \mathrm{e}^{-\mathrm{i} \alpha^{2} / \delta \epsilon}\right\} .
\end{aligned}
$$

The zeros of $D$, i.e. the poles for the Burgers solution, are solutions $\delta=x / t$ of the equation

$$
\cos \left(\frac{\delta}{2 \epsilon} \sqrt{\frac{\alpha}{2}}\right)=\frac{\mathrm{i}}{\sqrt{2}} \mathrm{e}^{\mathrm{i} \alpha^{2} / \delta \epsilon} .
$$

Note that, if $\delta$ is a solution of (5.10), then so is $-\delta$ and $\delta+\epsilon \alpha_{1} n$ with

$$
\alpha_{1}=4 \pi t \sqrt{\frac{2}{\alpha}} .
$$

The solutions of (5.10) are

$$
\delta=\delta_{n}^{ \pm}= \pm \epsilon\left(\left(x_{0}+\mathrm{i} y_{0}\right) \sqrt{\frac{8}{\alpha}}+n \alpha_{1}\right)
$$

and $\left(x_{0}, y_{0}\right)$ are a particular solution of the equations

$$
\begin{aligned}
& \sqrt{2} \cos \left(x_{0}\right) \cosh y_{0}=-\sin \left(\frac{\alpha^{2}}{8 \epsilon}\right), \\
& \sqrt{2} \sin \left(x_{0}\right) \sinh y_{0}=-\cos \left(\frac{\alpha^{2}}{8 \epsilon}\right) .
\end{aligned}
$$

One can easily show that there is a unique solution $\left(x_{0}, y_{0}\right)$ up to translation and reflection as in (5.12). This shows that to leading order the poles of the dispersive Burgers equation lie on two staggered horizontal linear arrays with spacing $\epsilon \alpha_{1}$. 




Figure 2. $\Im a_{j}(t, v)$ versus $\Re a_{j}(t, v)$. Time evolution in $\mathbb{C}$ of $a_{j}(t, v), j=-4, \cdots, 4$ for $v=10^{-5} \times \mathrm{i}$ and $N$ (number of poles) $=10^{5} . t_{\text {initial }}=t_{*}=1$ and $t_{\text {final }}=1.25$. Typical time step $\delta t \approx 10^{-3} \cdot n_{\text {steps }}=175$. Time-stepping tolerance: $10^{-8}<$ LRT $<10^{-4}$ (where LRT is local relative tolerance).

5.2. $x \in\left(-x_{s}(t)-\delta / 2, x_{s}(t)+\delta / 2\right)^{c}, \delta>0, v=\mathrm{i} \epsilon, \epsilon \rightarrow 0^{+}, t>t_{*}$

The inviscid limit is found in a straightforward manner in this case; only one saddle point is relevant, so that the asymptotic limit derived in section 5.1 reduces to

$$
\Psi_{\mathrm{i} \epsilon}(x, t)=\Psi(x, t)+\mathrm{O}(\epsilon) \quad \text { as } \epsilon \rightarrow 0^{+},
$$

where $\Psi(x, t)=z_{s}(x, t)$ is the spatially singular part of the dispersionless solution (see section 6 of $[29, \mathrm{I}]$ for more details). Thus the solution outside the caustic behaves according to the following property.

Property 5.2 As $v \rightarrow 0^{+}$for $x \in\left(-x_{s}(t)-\delta / 2, x_{s}(t)+\delta / 2\right)^{c}, \delta>0, t>t_{*}$, the solution to the Burgers equation is given by

$$
\begin{aligned}
& \Psi_{i \epsilon}(x, t)=\frac{x}{t}-\frac{\Psi_{\mathrm{i} \epsilon}(x, t)}{t}=\frac{x}{t}-\frac{\Psi(x, t)}{t}+\mathrm{O}(\epsilon) \quad \text { as } \epsilon \rightarrow 0^{+}, \\
& \Psi(x, t)=z_{s *}(x, t), \quad z_{s *}: \Re w\left(z_{s *}, x\right)=\max _{s=0,1,2} \Re w\left(z_{s}, x\right) .
\end{aligned}
$$

5.3. Uniform asymptotic expansion as $v \rightarrow 0$ for $t>t_{*}$ across the caustic $x= \pm x_{s}(t)$ via Pearcey integral

Following the notation of Kaminski [23], we introduce the Pearcey integral from which one can derive a uniform asymptotic expansion with two coalescing saddle points (see also [32]). Let

$$
P(X, Y)=\int_{-\infty}^{+\infty} \mathrm{e}^{\mathrm{i}\left(u^{4} / 4+X u^{2} / 2+Y u\right)} \mathrm{d} u
$$




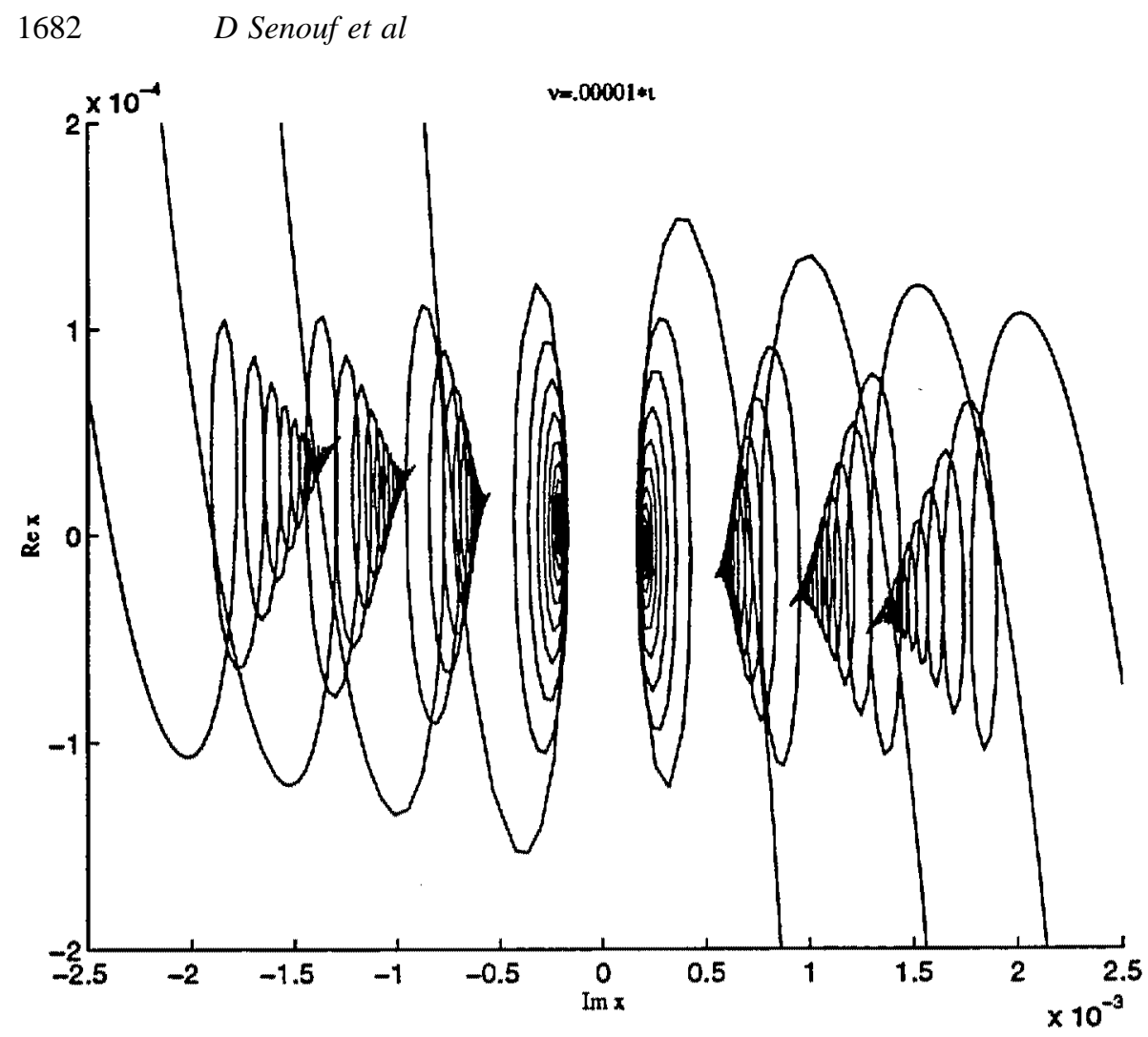

Figure 3. Close-up of figure 2 .

denote the Pearcey integral. In the arbitrary case $|\theta| \leqslant \pi / 2$, letting $y \rightarrow(-i v / 2)^{1 / 4} u=$ $e^{i 3 \pi / 8}(v / 2)^{1 / 4} u$, one can express $E_{\epsilon \mathrm{e}^{\mathrm{i} \theta}}(x, t)$ in terms of $P(X, Y)$

$E_{\epsilon \mathrm{e}^{\mathrm{i} \theta}}(x, t)=\left(\frac{\epsilon \mathrm{e}^{\mathrm{i} \theta}}{2 i}\right)^{1 / 4} P\left(X=\frac{\alpha}{\sqrt{2 \epsilon}} \mathrm{e}^{\mathrm{i}(\pi / 4-\theta / 2)}, Y=\frac{x}{2 t}\left(\frac{1}{2 \epsilon^{3}}\right)^{1 / 4} \mathrm{e}^{-\mathrm{i}(\pi / 8+3 \theta / 4)}\right)$.

In particular for $\theta=0(v=\epsilon \in \mathbb{R})$, we have (cf. equation $(3.12)$ in $[29, \mathrm{I}])$

$$
E_{\epsilon}(x, t)=\left(\frac{\epsilon}{2 \mathrm{i}}\right)^{1 / 4} P\left(X=\frac{\alpha}{\sqrt{2 \epsilon}} \mathrm{e}^{\mathrm{i} \pi / 4}, Y=\frac{x}{2 t}\left(\frac{1}{2 \epsilon^{3}}\right)^{1 / 4} \mathrm{e}^{-\mathrm{i} \pi / 8}\right) .
$$

For $\theta=\pi / 2(v=\mathrm{i} \epsilon)$, from (3.5) we can express $E_{\mathrm{i} \epsilon}(x, t)$ as

$$
\begin{aligned}
E_{\mathrm{i} \epsilon}(x, t)= & \int_{-\infty}^{+\infty} \exp \left\{\frac{\mathrm{i}}{2 \epsilon}\left(y^{4}-\alpha y^{2}-\frac{x}{t} y\right)\right\} \mathrm{d} y \\
& =\left(\frac{\epsilon}{2}\right)^{1 / 4} \int_{-\infty}^{+\infty} \exp \left\{\mathrm{i}\left(\frac{u^{4}}{4}-\frac{\alpha}{\sqrt{2 \epsilon}} \frac{u^{2}}{2}-\frac{x}{2 t}\left(\frac{1}{2 \epsilon^{3}}\right)^{1 / 4} u\right)\right\} \mathrm{d} u \\
& =\left(\frac{\epsilon}{2}\right)^{1 / 4} P\left(X(\epsilon ; t)=\frac{-\alpha}{\sqrt{2 \epsilon}}, Y(\epsilon ; x, t)=\frac{-x}{2 t}\left(\frac{1}{2 \epsilon^{3}}\right)^{1 / 4}\right) .
\end{aligned}
$$

Clearly a small $\epsilon$ asymptotic of $E_{\mathrm{i} \epsilon}$ is equivalent to a combined asymptotic expansion of the Pearcey integral as $|X|,|Y| \rightarrow+\infty$. The caustic of $P(X, Y)$ and the corresponding 


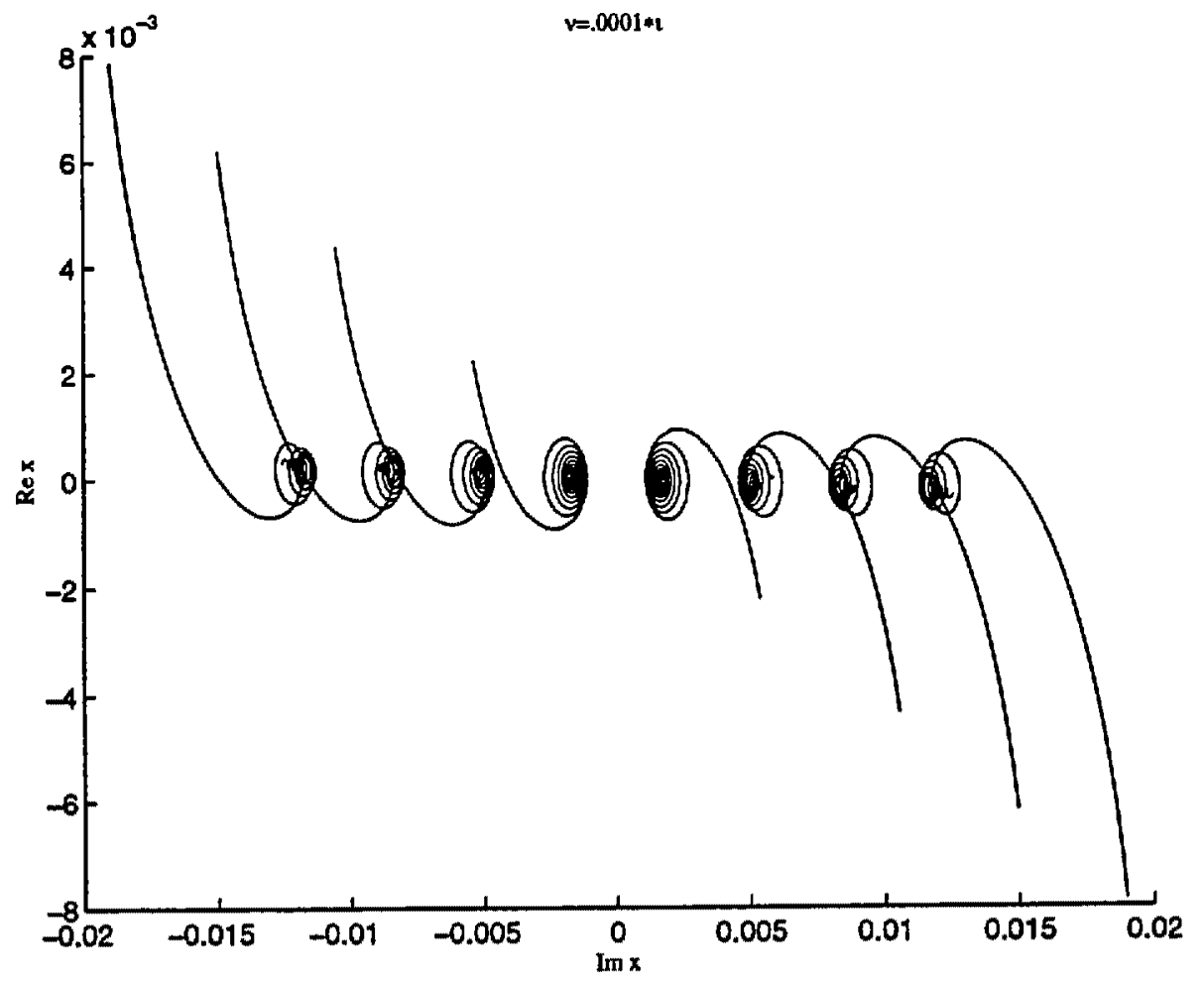

Figure 4. $\Im a_{j}(t, v)$ versus $\Re a_{j}(t, v)$. Time evolution in $\mathbb{C}$ of $a_{j}(t, v), j=-4, \cdots, 4$ for $v=10^{-4} \mathrm{i}$ and $N=10^{5} . t_{\text {initial }}=t_{*}=1$ and $t_{\text {final }}=2 . n_{\text {steps }}=280$. Typical time step $\delta t \approx 3 \times 10^{-3}$. Time-stepping tolerance: $10^{-8}<$ LRT $<10^{-4}$.

caustic of $E_{\mathrm{i} \epsilon}(x, t)$ is given by

$$
Y=\frac{2}{\sqrt{27}}(-X)^{3 / 2} \Longleftrightarrow x= \pm x_{s}(t) \in \mathbb{R} \quad \text { for } t>t_{*} .
$$

Hence the uniform asymptotic behaviour of $E_{\mathrm{i} \epsilon}$ in a neighbourhood of the caustic is found from the one of $P\left(-X,(2 / \sqrt{27}-\tau) X^{3 / 2}\right)$ as $X \rightarrow+\infty$, where $\tau=0$ at the caustic, and $\tau \neq 0$ away from it [23]. This amounts to a uniform expansion valid for $\left|x \pm x_{s}(t)\right| \leqslant\left|\delta_{ \pm}(\tau ; t)\right|$ where $\delta_{ \pm}(\tau)=\delta_{ \pm}(\tau ; t)=\mp(\sqrt{27} / 2)\left|x_{s}(t)\right| \tau$. This expansion is also valid outside of these intervals centred about $\pm x_{s}(t)$; however, the region of interest is the neighbourhood of the caustic. Indeed one only needs to use the asymptotic expansion of the Airy function and its derivative to find the results obtained in sections 5.1 and 5.2.

From (3.11) and (5.14) we have that

$$
\begin{aligned}
\Psi_{\mathrm{i} \epsilon}(x, t) & =t 2 \mathrm{i} \epsilon \partial_{x} \log \left(E_{i \epsilon}(x, t)\right) \\
& =t 2 \mathrm{i} \epsilon \partial_{x} \log \left[P\left(X(\epsilon ; t)=\frac{-\alpha}{\sqrt{2 \epsilon}}, Y(\epsilon ; x, t)=\frac{-x}{2 t}\left(\frac{1}{2 \epsilon^{3}}\right)^{1 / 4}\right)\right] .
\end{aligned}
$$

Let

$$
X=X(\epsilon ; t), \quad Y=Y(\epsilon ; t)=Y\left(\epsilon ; x= \pm x_{s}(t)-\delta_{ \pm}(\tau ; t), t\right),
$$




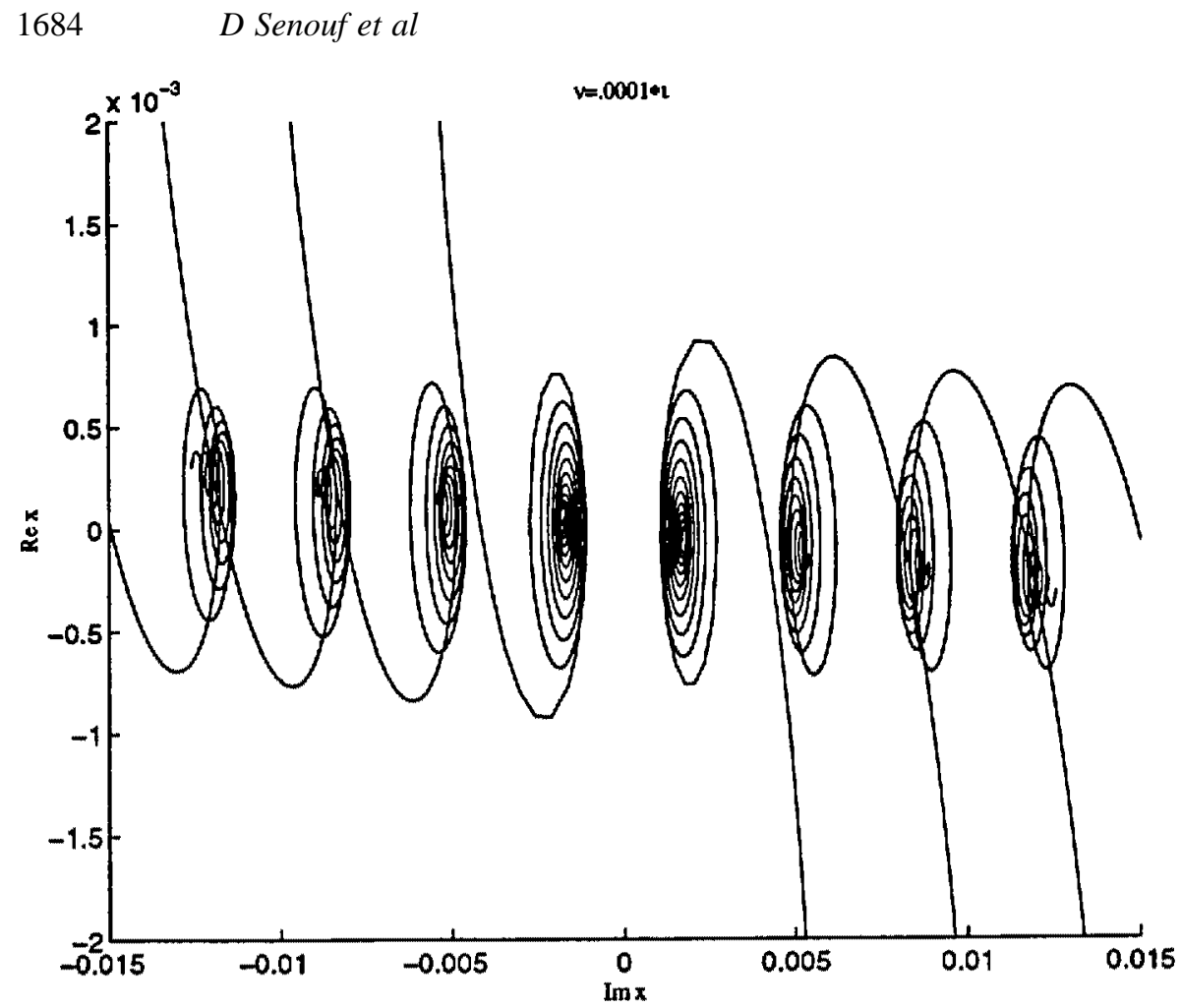

Figure 5. Close-up of figure 4.

where $\delta \pm(\tau ; t) \rightarrow 0$ as $\tau \rightarrow 0$, so that $\Psi_{\mathrm{i} \epsilon}\left(x= \pm x_{s}(t)-\delta_{ \pm}(\tau ; t), t\right)=t 2 \mathrm{i} \epsilon \partial_{x} \log (P(X, Y))$

$$
=-t 2 \mathrm{i} \epsilon \partial_{\tau} \log \left(P\left(-X,(2 / \sqrt{27}-\tau) X^{3 / 2}\right)\right) / \frac{\partial \delta_{ \pm}}{\partial \tau} .
$$

Let $P(\tau)=P\left(-X,(2 / \sqrt{27}-\tau) X^{3 / 2}\right)$ then, since $\partial \delta_{ \pm} / \partial \tau=\mp \sqrt{27} x_{s}(t) / 2$, we have

$$
\Psi_{\mathrm{i} \epsilon}\left(x= \pm x_{s}(t)-\delta_{ \pm}(\tau ; t), t\right)= \pm \frac{4 t i \epsilon}{\sqrt{27} x_{s}(t)} \frac{P_{\tau}(\tau)}{P(\tau)} .
$$

Following the notation presented in [29], let

$p_{0}(\tau)=3^{-1 / 6}(1+\mathrm{O}(\tau)), \quad q_{0}(\tau)=-\frac{3^{-5 / 6}}{2}(1+\mathrm{O}(\tau)), \quad \zeta(\tau)=3^{-1 / 6} \tau(1+\mathrm{O}(\tau))$,

and

$$
f(v)=f(v ; \tau)=\frac{v^{4}}{4}-\frac{v^{2}}{2}+\left(\frac{2}{\sqrt{27}}-\tau\right) v,
$$

and the $v_{i}, i=1,2,3$ are the saddle points of $f(v ; \tau)$ determined by the equation $f_{v}\left(v_{i} ; \tau\right)=0$, so that $f\left(v_{i} ; \tau\right)=-v_{i}^{2} / 4+(2 / \sqrt{27}-\tau) 3 v_{i} / 4$. The $v_{i}$ are specifically

$$
\begin{array}{ll}
v_{1}(\tau) & =-\frac{2}{\sqrt{3}} \sin \left(\frac{\pi}{3}+\phi(\tau)\right), \quad v_{2}(\tau)=\frac{2}{\sqrt{3}} \sin (\phi(\tau)), \\
v_{3}(\tau) & =\frac{2}{\sqrt{3}} \sin \left(\frac{\pi}{3}-\phi(\tau)\right),
\end{array}
$$




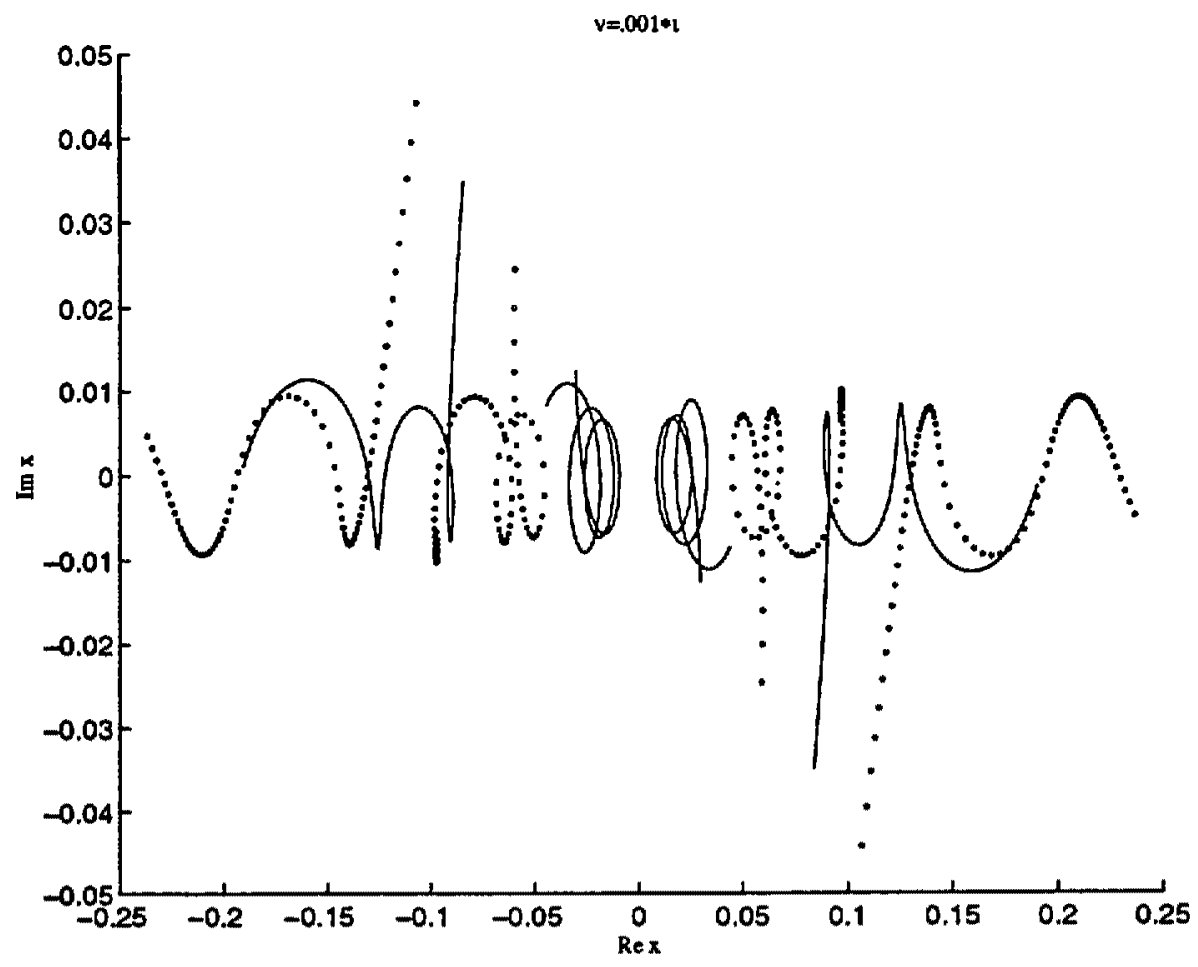

Figure 6. $\Im a_{j}(t, v)$ versus $\Re a_{j}(t, v)$. Time evolution in $\mathbb{C}$ of $a_{j}(t, v), j=-4, \cdots, 4$ for $v=10^{-3} \mathrm{i}$ and $N=10^{5} . t_{\text {initial }}=t_{*}=1$ and $t_{\text {final }}=4 . n_{\text {steps }}=80$. Typical time step $\delta t=0.05$. Time-stepping tolerance: $10^{-8}<$ LRT $<10^{-4}$.

where

$$
\phi=\phi(\tau)=\frac{1}{3} \arcsin (1-\tau \sqrt{27} / 2), \quad \tau \in \mathbb{R}, \quad|\phi| \leqslant \frac{\pi}{6} .
$$

Since

$$
X=\frac{-\alpha}{\sqrt{2 \epsilon}} \Rightarrow \frac{\mathrm{i} X^{2}}{2}=\mathrm{i} \frac{\alpha^{2}}{4 \epsilon} \Rightarrow X^{-2}=\mathrm{O}(\epsilon)
$$

then according to the expansion of the Pearcey integral presented by Kaminski [23], we have proved the following property.

Property 5.3 The uniform asymptotic expansion as $\epsilon \rightarrow 0^{+}$of $\Psi_{\mathrm{i} \epsilon}\left(x= \pm x_{s}(t)-\delta_{ \pm}(\tau ; t), t\right)$ in a neighbourhood of the caustics $x= \pm x_{s}(t)$ is

$$
\begin{aligned}
\Psi_{\mathrm{i} \epsilon}\left(x= \pm x_{s}(\right. & \left.t)-\delta_{ \pm}(\tau ; t), t\right)= \pm \frac{\sqrt{3}}{2}\left(\frac{x_{s}(t)}{t}\right)^{1 / 3}\left[\left[v_{2}+v_{3}\right] e^{i \frac{\alpha^{2}}{4 \epsilon}}\left[f\left(v_{2}\right)+f\left(v_{3}\right)\right]\right. \\
\times & \left\{p_{0}(\tau) \frac{2 \pi}{X^{1 / 6}} A \mathrm{i}\left(-X^{4 / 3} \zeta(\tau)\right)+q_{0}(\tau) \frac{2 \pi}{\mathrm{i} X^{5 / 6}} A \mathrm{i}^{\prime}\left(-X^{4 / 3} \zeta(\tau)\right)\right\} \\
+ & \left.2 v_{1} \mathrm{e}^{\mathrm{i} \frac{\alpha^{2}}{4 \epsilon} 2 f\left(v_{1}\right)}\left(\frac{\pi}{3 v_{1}^{2}-1}\right)^{1 / 2} \frac{1+\mathrm{i}}{X^{1 / 2}}\right] \\
& /\left[\mathrm { e } ^ { \mathrm { i } \frac { \alpha ^ { 2 } } { 4 \epsilon } [ f ( v _ { 2 } ) + f ( v _ { 3 } ) ] } \left\{p_{0}(\tau) \frac{2 \pi}{X^{1 / 6}} A \mathrm{i}\left(-X^{4 / 3} \zeta(\tau)\right)\right.\right.
\end{aligned}
$$




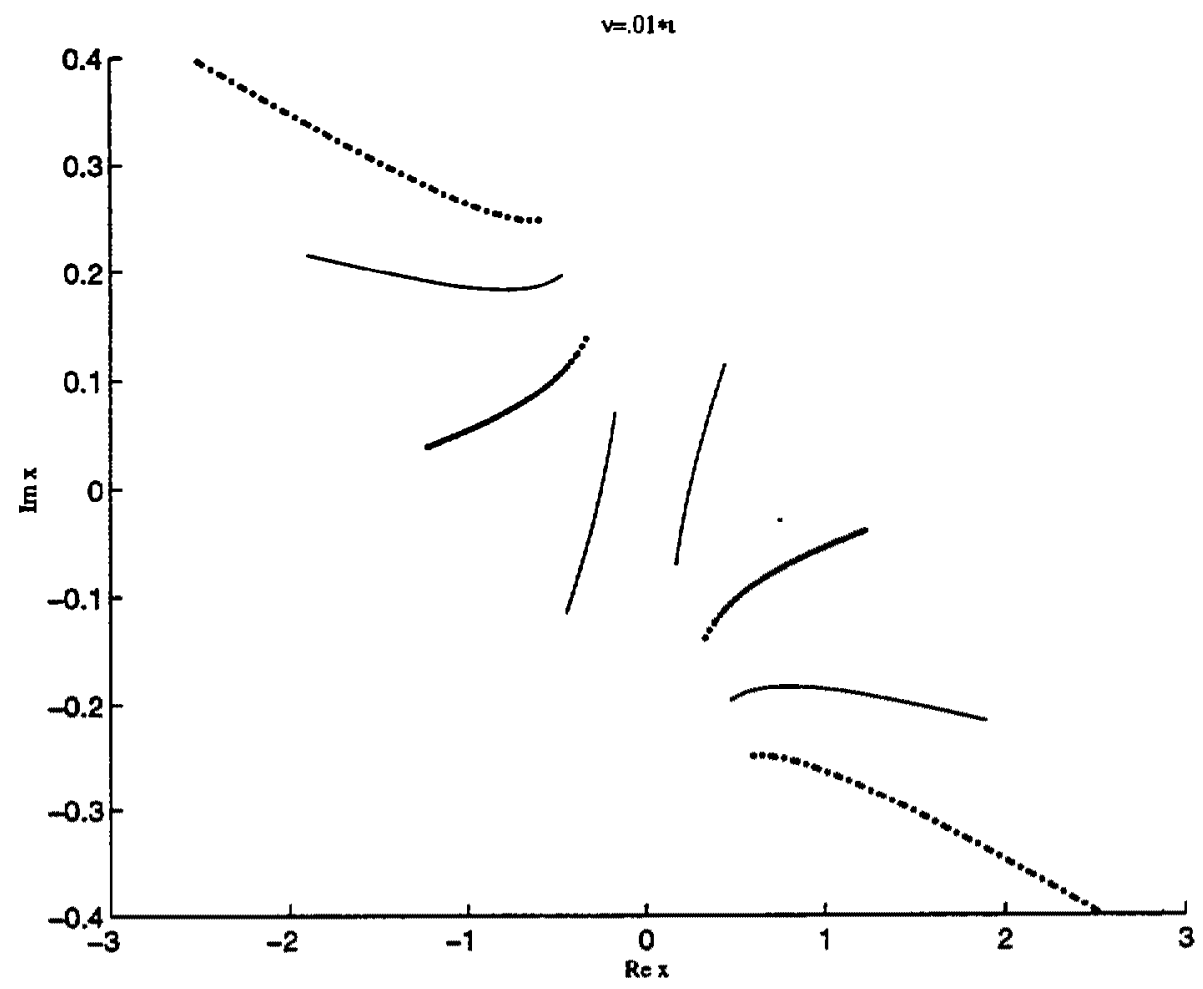

Figure 7. $\Im a_{j}(t, v)$ versus $\Re a_{j}(t, v)$. Time evolution in $\mathbb{C}$ of $a_{j}(t, v), j=-4, \cdots, 4$ for $v=10^{-2} \mathrm{i}$ and $N=10^{5} . t_{\text {initial }}=t_{*}=1$ and $t_{\text {final }}=5 . n_{\text {steps }}=48$. Typical time step $\delta t=0.1$. Time-stepping tolerance: $10^{-8}<$ LRT $<10^{-4}$.

$$
\begin{aligned}
& \left.\left.+q_{0}(\tau) \frac{2 \pi}{\mathrm{i} X^{5 / 6}} A \mathrm{i}^{\prime}\left(-X^{4 / 3} \zeta(\tau)\right)\right\}+\mathrm{e}^{\mathrm{i} \frac{\alpha^{2}}{4 \epsilon} 2 f\left(v_{1}\right)}\left(\frac{\pi}{3 v_{1}^{2}-1}\right)^{1 / 2} \frac{1+\mathrm{i}}{X^{1 / 2}}\right] \\
& +\mathrm{O}(\epsilon) \text { as } \epsilon \rightarrow 0^{+}
\end{aligned}
$$

5.3.1. Behaviour at the caustics $x= \pm x_{s}(t) \quad$ At the caustics $x= \pm x_{s}(t), \tau=0$,

$\phi(0)=\pi / 6, \quad v_{1}(0)=-2 / \sqrt{3}, \quad v_{2}(0)=v_{3}(0)=1 / \sqrt{3}$,

$f\left(v_{i} ; 0\right)=-v_{i}^{2} / 4+v_{i} / 2 \sqrt{3}, \quad f\left(v_{2} ; 0\right)=f\left(v_{3} ; 0\right)=-2 / 3, \quad f\left(v_{1} ; 0\right)=1 / 12$.

Since $X=\mathrm{O}\left(\epsilon^{-1 / 2}\right)$, the dominant term as $\epsilon \rightarrow 0^{+}$in both the numerator and the denominator of $\Psi_{\mathrm{i} \epsilon}$ is obviously the term containing the factor $X^{-1 / 6}$. Therefore the dominant behaviour of $\Psi_{\mathrm{i} \epsilon}\left( \pm x_{s}(t), t\right)$ reduces to the simple form

$$
\begin{aligned}
\Psi_{i \epsilon}\left( \pm x_{s}(t), t\right) & = \pm \frac{\sqrt{3}}{2}\left(\frac{x_{s}(t)}{t}\right)^{1 / 3}\left(v_{2}(0)+v_{3}(0)\right)+\mathrm{O}(\epsilon) \\
& = \pm\left(\frac{x_{s}(t)}{t}\right)^{1 / 3}+\mathrm{O}(\epsilon) \text { as } \epsilon \rightarrow 0^{+} .
\end{aligned}
$$





Figure 8. $\Re \psi_{v}(x, t)$ versus $x$ from the pole dynamics for $v=10^{-4} \mathbf{i}$ and $N=10^{5}$ at $t=1,1.05,1.1,1.15$.

\section{Continuum limit of the pole expansion and the Calogero dynamical system}

From the equation for the pole dynamics and the Mittag-Leffler expansion of the non-zero dispersion solution, one can obtain a set of equations for the inviscid limit which give a new representation of the solution to the inviscid Burgers equation. Recall the pole expansion

$$
\psi_{v}(x, t)=\frac{x}{t}-\sum_{n=1}^{\infty} \frac{4 v x}{x^{2}-a_{n}^{2}(t, v)}=\frac{x}{t}-2 v \sum_{\substack{n=-\infty \\ n \neq 0}}^{\infty} \frac{1}{x-a_{n}},
$$

and the pole dynamics; $\forall n \in \mathbb{N}^{*}$,

$$
\dot{a}_{n}=\frac{a_{n}}{t}-\frac{v}{a_{n}}-4 v a_{n} \sum_{\substack{l=1 \\ l \neq n}}^{\infty} \frac{1}{a_{n}^{2}-a_{l}^{2}}=\frac{a_{n}}{t}-2 v \sum_{\substack{l=-\infty \\ l \neq n, 0}}^{\infty} \frac{1}{a_{n}-a_{l}} .
$$

Define the complex map $\mathcal{F}(\zeta, v, t)$ as

$$
a_{n}(t, v)=\mathcal{F}\left(\zeta_{n}^{v}=v n, v, t\right): \mathbb{Z}^{*} \times \mathbb{R}^{+} \times \mathbb{R}^{+} \rightarrow \mathbb{C}, \quad a_{-n}=-a_{n} .
$$

At $t_{*}$, we have [29 (I, section 4.1)]

$$
\begin{aligned}
a_{n}\left(t_{*}, v\right) & =\mathcal{F}\left(\zeta_{n}^{v}=v n, v, t_{*}\right)=\mathrm{i} 4 t_{*}\left(2 v \mu_{n}\right)^{3 / 4} \\
& =\mathrm{i} 4 t_{*}\left(2 v\left(c_{-1} n+c_{0}+c_{1} / n+\cdots\right)\right)^{3 / 4} \\
& \left.=\mathrm{i} 4 t_{*}\left(c_{-1}(2 v n)+c_{0} 2 v+c_{1}(2 v)^{2} /(2 v n)+\cdots\right)\right)^{3 / 4} .
\end{aligned}
$$



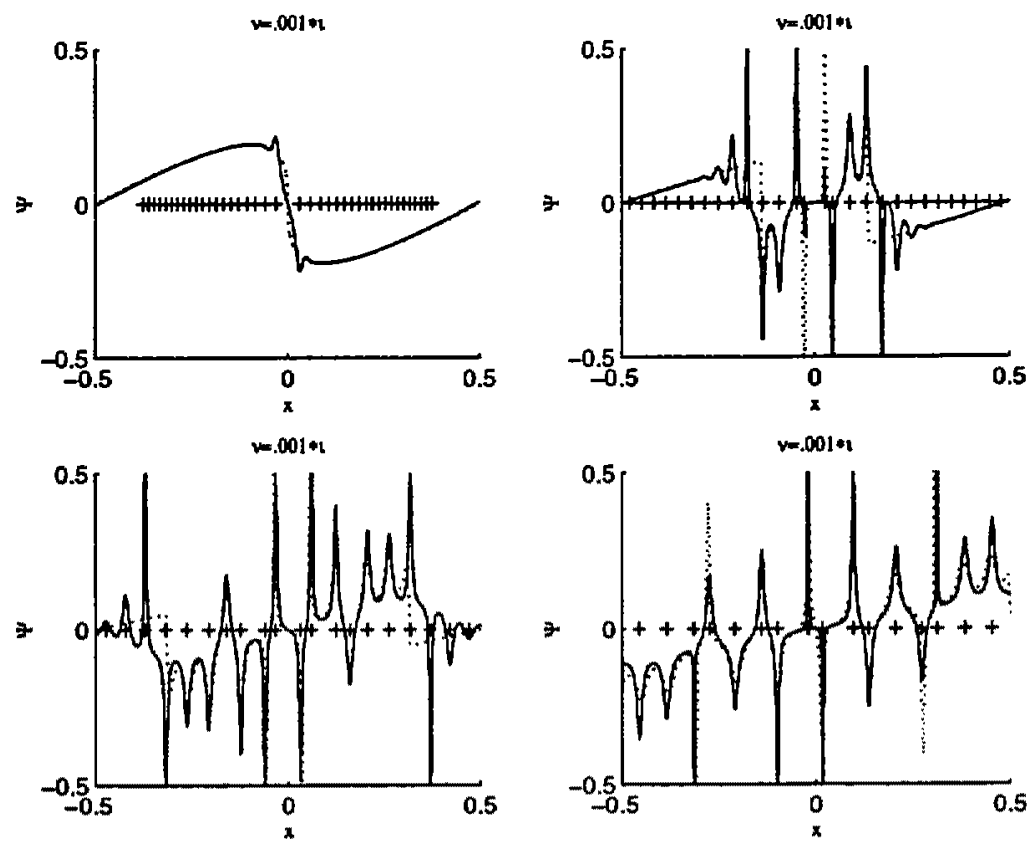

Figure 9. $\Re \Psi_{v}(x, t)$ versus $x$. Comparison of pole dynamics $(-)$ and stationary phase approximation $(\cdots \cdots)$ for $v=10^{-3} \mathrm{i}$ and $N=10^{5}$. Projection of pole locations on the real axis (+) at $t=1,2,3,4$.

Then introduce the map

$$
f(\zeta, t)=\mathcal{F}(\zeta, 0, t): \mathbb{R} \times \mathbb{R}^{+} \rightarrow \mathbb{C}, \quad f(-\zeta, t)=-f(\zeta, t),
$$

where the continuous variable $\zeta$ corresponds to a position on the real axis which can be thought of as a variable obtained by simultaneously letting $v \rightarrow 0^{+}$and $n \rightarrow+\infty$. Assume that

$$
a_{n}(t, v)=\mathcal{F}(n|v|, v, t)=f(n|v|, t)+e_{n}(t, v)
$$

in which $e_{n}(t, v)$ is a small error term that goes to 0 as $v \rightarrow 0$. Now let $|v| \rightarrow 0$ so that $\eta=v /|v|$ remains constant. Then, at least formally,

$$
\begin{aligned}
2 v \sum_{\ell \neq n} \frac{1}{a_{n}(t, v)-a_{\ell}(t, v)} & \simeq 2 \eta|v| \sum_{\ell \neq n} \frac{1}{f(n|v|, t)-f(\ell|v|, t)} \\
& \stackrel{v \rightarrow 0}{\longrightarrow} 2 \eta \mathrm{PV} \int_{-\infty}^{\infty} \frac{\mathrm{d} \zeta^{\prime}}{f(\zeta, t)-f\left(\zeta^{\prime}, t\right)} .
\end{aligned}
$$

Moreover, this approximation shows that the representation (6.4) is valid for all time if it is true at $t=0$. A rigorous analysis of the approximation (6.5) has been performed in the context of vortex sheets in [9]. A rigorous justification of this limiting process is also presented by other means in [29, II] for the real viscosity case. It is then clear that the pair of equations $(6.1 a)$ and $(6.1 b)$ satisfy the following:

Property 6.1 The continuum limit of the Calogero dynamical system and the pole expansion is the system of integrodifferential equations defined for any $x$ such that $\forall \zeta \in \mathbb{R}, x \neq f(\zeta, t)$, 


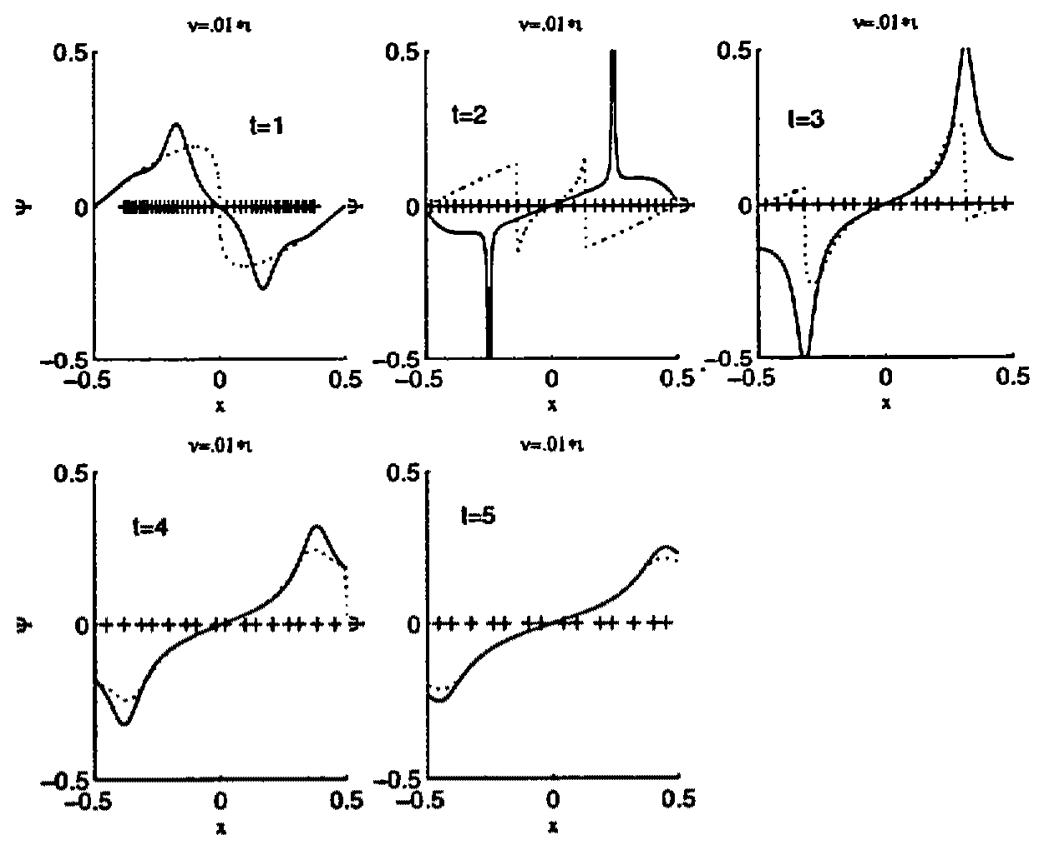

Figure 10. $\Re \psi_{v}(x, t)$ versus $x$. Comparison of pole dynamics $(-)$ and stationary phase approximation $(\cdots \cdots)$ for $v=10^{-2} \mathrm{i}$ and $N=10^{5}$ at $t=1,2,3,4,5$. Projection of pole locations on the real axis $(+)$.

by

$$
\begin{aligned}
& \frac{\partial f}{\partial t}(\zeta, t)=\frac{f(\zeta, t)}{t}-2 \eta \mathrm{PV} \int_{-\infty}^{\infty} \frac{\mathrm{d} \zeta^{\prime}}{f(\zeta, t)-f\left(\zeta^{\prime}, t\right)}, \\
& \psi(x, t)=\frac{x}{t}-2 \eta \int_{-\infty}^{\infty} \frac{\mathrm{d} \zeta^{\prime}}{x-f\left(\zeta^{\prime}, t\right)} .
\end{aligned}
$$

This property can also be expressed as

$$
\begin{aligned}
\frac{\partial f}{\partial t}(\zeta, t) & =\frac{f(\zeta, t)}{t}-2 \eta f(\zeta, t) \int_{0}^{\infty} \frac{\mathrm{d} \zeta^{\prime}}{f^{2}(\zeta, t)-f^{2}\left(\zeta^{\prime}, t\right)} \\
& =\frac{f(\zeta, t)}{t}-\eta f(\zeta, t) \operatorname{PV} \int_{-\infty}^{\infty} \frac{\mathrm{d} \zeta^{\prime}}{f^{2}(\zeta, t)-f^{2}\left(\zeta^{\prime}, t\right)}
\end{aligned}
$$

and

$$
\begin{aligned}
\psi(x, t) & =\frac{x}{t}-2 \eta x \int_{0}^{\infty} \frac{\mathrm{d} \zeta^{\prime}}{x^{2}-f^{2}\left(\zeta^{\prime}, t\right)} \\
& =\frac{x}{t}-\eta x \int_{-\infty}^{\infty} \frac{\mathrm{d} \zeta^{\prime}}{x^{2}-f^{2}\left(\zeta^{\prime}, t\right)}, \quad x \neq f(\zeta, t) .
\end{aligned}
$$

The system consisting of equations $(6.6 a)$ and $(6.6 b)$ provides a slowly varying, but incomplete, description of the solution of the Burgers equation and of the pole dynamics. Let $f(\zeta, t)$ solve the continuum (i.e. slowly varying) equation (6.6a). Then the approximate pole positions are given by (6.4) and the solution $\psi_{\nu}$ of the Burgers equation by the pole expansion (6.1a). Furthermore, as shown in the next section, the corresponding solution $\psi$ of the inviscid Burgers equation is given by $(6.6 b)$, and the image of $f$ in $\mathbb{C}$ is a 

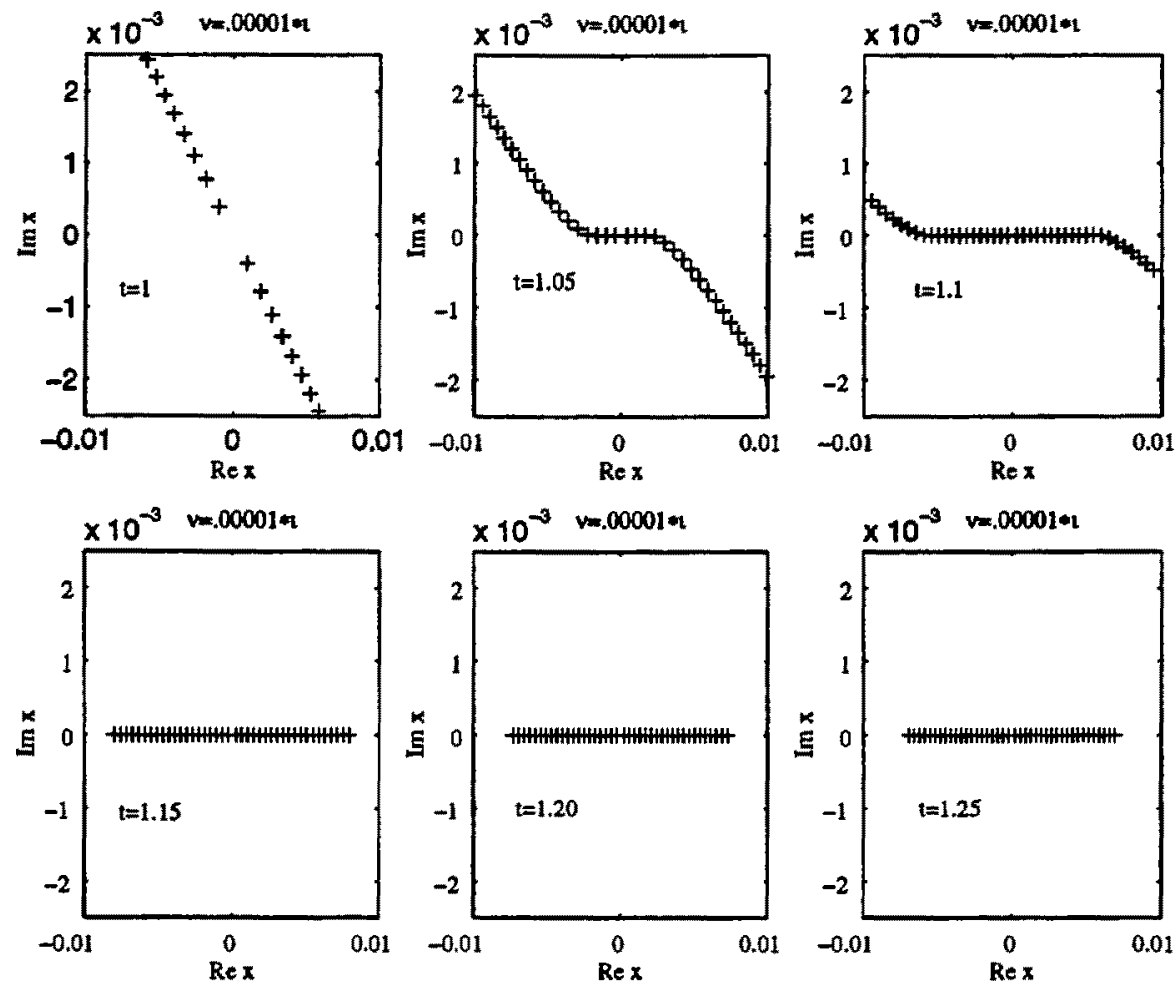

Figure 11. Branch-cut dynamics for $v=10^{-5} \mathrm{i}$ at $t=1,1.05,1.1,1.15,1.2,1.25$.

branch cut for $\psi$. This is an incomplete description, since it does not yield a formula for the wavelength, phase and amplitude of the oscillations. Moreover, when the poles are close to the real line, the oscillations in the Burgers solution are quite sensitive to small errors. In fact, computations presented in section 7.3 for $v=\epsilon i$, show that some of the poles found through this 'branch-cut dynamics' method lie on the real axis, which makes the reconstruction of the solution $\psi_{v}$ impossible. We believe that this difficulty could be overcome through improvements in the approximation (6.5).

\subsection{Branch-cut dynamics}

The branch-cut dynamics method, presented in this section, is a new method for solving the inviscid Burgers equation

$$
\psi_{t}+\psi \psi_{x}=0
$$

The main interest here in this method is that it represents the continuum limit, as $|\nu| \rightarrow 0$, of the pole dynamics for the viscous equation. It is also interesting to note that the resulting integro-differential equation is nearly the same as the Birkhoff-Rott equation for a vortex sheet, but without the complex conjugation on the right-hand side. The branch-cut dynamics have a parametric and a non-parametric formulation. In the parametric formulation, the solution is described through the dynamics of a complex-valued function $f(\zeta, t)$ of a real 



Figure 12. Branch-cut dynamics for $v=10^{-4} \mathrm{i}$ at $t=1,1.1,1.2,1.3$.

variable $\zeta$. Let $f(\zeta, t)$ satisfy

$$
f_{t}(\zeta, t)=\frac{f(\zeta t)}{t}-2 \eta \mathrm{PV} \int_{-\infty}^{\infty} \frac{\mathrm{d} \zeta^{\prime}}{f(\zeta, t)-f\left(\zeta^{\prime}, t\right)}
$$

in which $\eta$ is an arbitrary constant. The integral is a Cauchy principal-value integral, due to the singularity at $\zeta^{\prime}=\zeta$, as well as possible singularities at $\zeta^{\prime}= \pm \infty$. Next define $\psi(x, t)$ by

$$
\psi(x, t)=\frac{x}{t}-2 \eta \int_{-\infty}^{\infty} \frac{\mathrm{d} \zeta^{\prime}}{x-f\left(\zeta^{\prime}, t\right)} .
$$

A straightforward calculation shows that $\psi(x, t)$ is a solution to the inviscid Burgers equation

$$
\psi_{t}+\psi \psi_{x}=0
$$

for any choice of $\eta$. These equations can be rephrased in a second non-parametric formulation involving a moving curve $\Gamma(t)$ in the complex plane (which may consist of several disconnected parts) and a density function $\rho(z, t)$ defined for $z \in \Gamma(t)$. In particular, $\Gamma(t)$ is the image of $f(\zeta, t)$ for $\zeta$ varying over the real line. The density function $\rho(z, t)$ is defined by [29 (II, section 5)]

$$
\rho(z, t)=\frac{1}{f_{\zeta}(\zeta, t)},
$$

in which $z=f(\zeta, t)$. Then $\mathrm{d} \zeta^{\prime}=\rho\left(z^{\prime}, t\right) \mathrm{d} z^{\prime}$ and

$$
\psi(x, t)=\frac{x}{t}-2 \eta \int_{\Gamma(t)} \frac{\rho\left(z^{\prime}, t\right)}{x-z^{\prime}} \mathrm{d} z^{\prime} .
$$



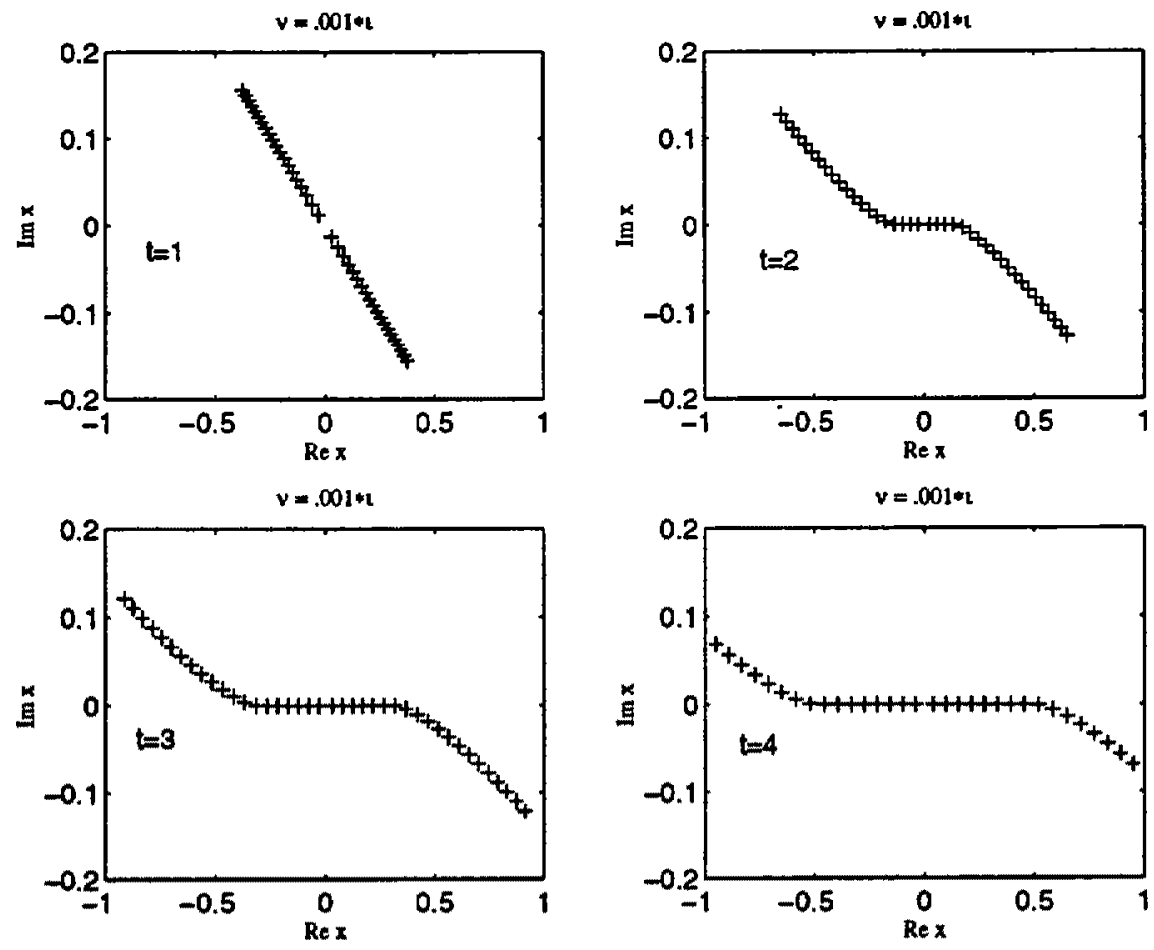

Figure 13. Branch-cut dynamics for $v=10^{-3} \mathrm{i}$ at $t=1,2,3,4$.

This formula can be extended into the complex $x$-plane but is discontinuous across the curve $\Gamma(t)$, i.e. $\Gamma(t)$ is a branch cut for the function $\psi$. Variations in the arbitrary complex parameter $\eta$ correspond to variations in the branch cut $\Gamma(t)$ for $\psi$, without change in the branch point. An application of the Plemejl formulae (see [14]) at a point $z$ on $\Gamma(t)$ shows that limiting values $\psi_{+}$and $\psi_{-}$from the right and left, respectively, are

$$
\psi_{ \pm}(z, t)=\frac{z}{t}-2 \eta \lim _{r \rightarrow 0} \int_{\Gamma_{ \pm}^{r}(t)} \frac{\rho\left(z^{\prime}, t\right)}{z-z^{\prime}} \mathrm{d} z^{\prime} \mp 2 \eta \pi \mathrm{i} \rho(z, t) .
$$

Here, the $\Gamma_{ \pm}^{r}(t)$ are contours identical to $\Gamma(t)$ except for removed semi-circular arcs of radius $r$ above and below the singularity. Since $\lim _{r \rightarrow 0} \Gamma_{ \pm}^{r}(t)=\Gamma(t)$, it follows that the difference in $\psi_{ \pm}$is

$$
\psi_{-}(z, t)-\psi_{+}(z, t)=4 \eta \pi \mathrm{i} \rho(z, t),
$$

and the average of $\psi_{ \pm}$is

$$
\begin{aligned}
\tilde{\Psi}(z, t) & \equiv \frac{1}{2}\left(\psi_{+}(z, t)+\psi_{-}(z, t)\right) \\
& =\frac{z}{t}-2 \eta \int_{\Gamma(t)} \frac{\rho\left(z^{\prime}, t\right)}{z-z^{\prime}} \mathrm{d} z^{\prime} \\
& =\frac{x}{t}-2 \eta \mathrm{PV} \int_{-\infty}^{\infty} \frac{\mathrm{d} \zeta^{\prime}}{f(\zeta, t)-f\left(\zeta^{\prime}, t\right)}=\frac{\partial f}{\partial t}(\zeta, t)
\end{aligned}
$$



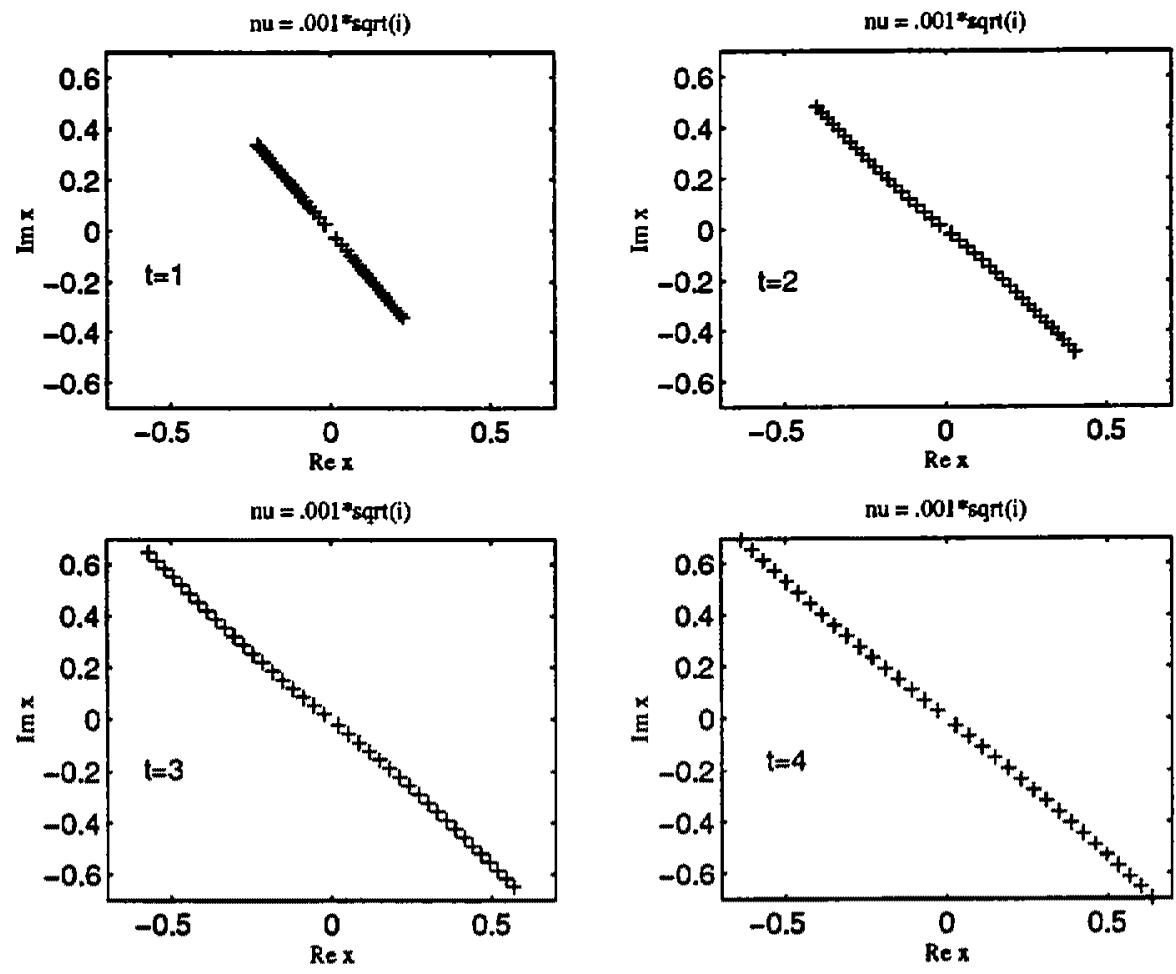

Figure 14. Branch-cut dynamics for $v=10^{-3} \mathrm{e}^{\mathrm{i} \pi / 4}$ at $t=1,2,3,4$.

Since $0=\psi_{t}+\psi \psi_{z}=\psi_{t}+\left(\frac{1}{2} \psi^{2}\right)_{z}$ for both $\psi=\psi_{+}$and $\psi_{-}$, it follows that $\rho$ satisfies the conservation equation

$$
\rho_{t}+(\tilde{\Psi} \rho)_{z}=0 .
$$

Therefore the branch-cut dynamics equations (6.8) and (6.9) are equivalent to the motion of $\Gamma(t)$ by the velocity $\tilde{\Psi}(z, t)$, and the evolution of the density $\rho(z, t)$ through $(6.16)$.

The usefulness of this method in the present context is its relation to the pole dynamics for the viscous (or dispersive) Burgers equation. An interesting equivalent form of the branch-cut dynamics equation (6.8) is found by considering the change of time variable

$$
\begin{array}{ll}
\tau \quad & =t^{-1}-t_{0}^{-1} \\
g(\zeta, \tau) & =t^{-1} f(\zeta, t)
\end{array}
$$

for any constant $t_{0}$. The resulting equation for $g$ is

$$
\frac{\partial g}{\partial \tau}(\zeta, \tau)=2 \eta \mathrm{PV} \int_{-\infty}^{\infty} \frac{\mathrm{d} \zeta^{\prime}}{g(\zeta, \tau)-g\left(\zeta^{\prime}, \tau\right)} .
$$

If $\eta=1 /(4 \pi \mathrm{i})$, and if the left-hand side was replaced by its complex conjugate $\partial \bar{g} / \partial \tau$, this equation would be identical to the Birkhoff-Rott equation for a vortex sheet [9].

\section{Numerics}

We present numerics which pertain to the analysis previously derived. That is, we use both the stationary phase formula and the pole dynamics as a means to compute the solution. A 

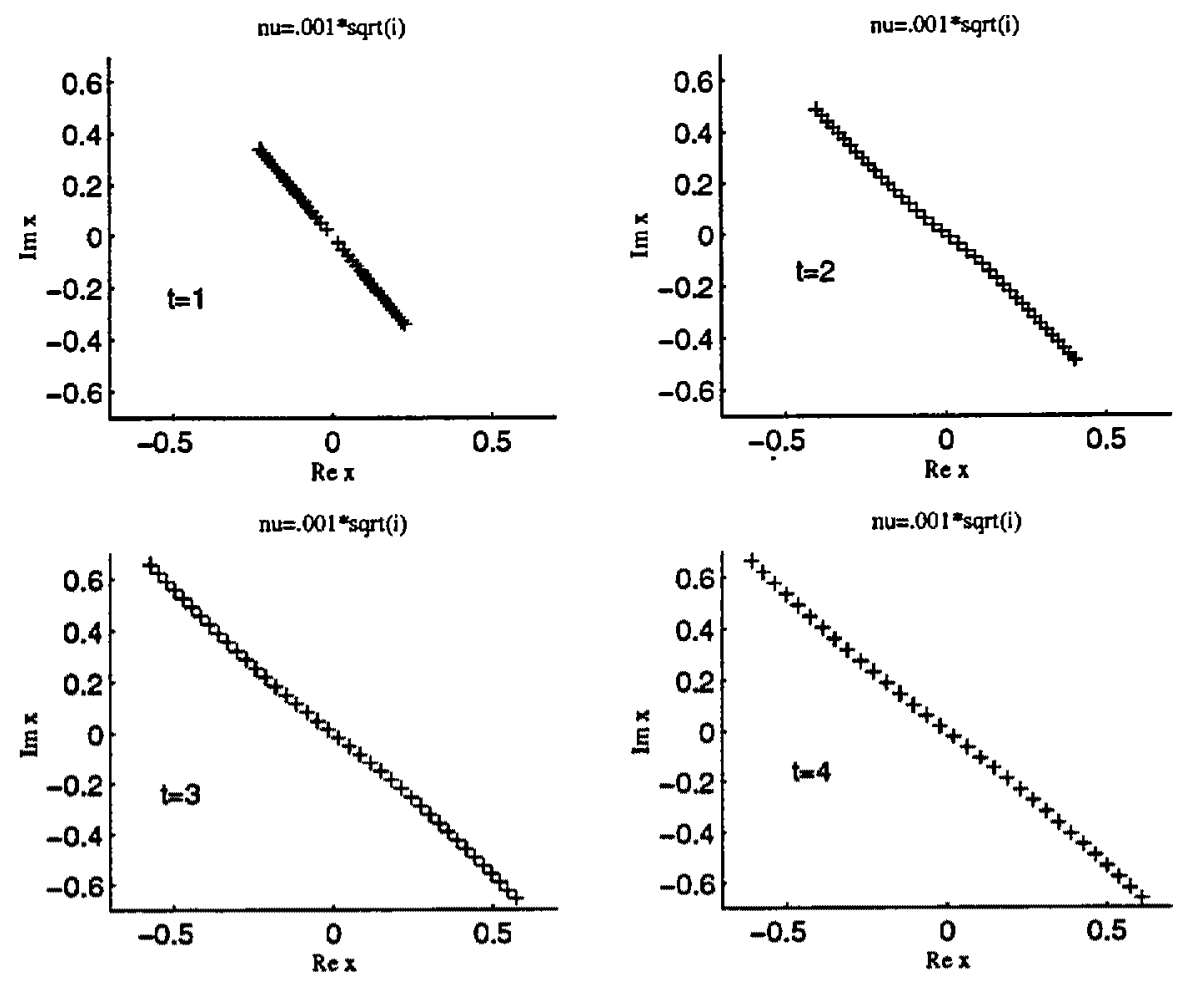

Figure 15. Positions of the first 20 poles from the pole dynamics for $v=10^{-3} \mathrm{e}^{\mathrm{i} \pi / 4}$ and $N=10^{5}$ at $t=1,2,3,4$.

third method based on a full finite-difference scheme is also presented initially. For all three methods, we set the parameter value $t_{*}=1$. In all the figures describing the behaviour of the solution $\psi_{v}$, we plot only the real part of the solution $\Re \psi_{v}$. Thus, whenever there is a label $\psi$, it should be understood as $\Re \psi_{\nu}$.

\subsection{Finite differences, Runge-Kutta scheme and pole expansion}

We present a numerical scheme which enables us to solve (3.1) for arbitrary values of $\arg v$ for moderately small values of $|\nu|=\epsilon$. The procedure is sometimes referred to as the method of lines and consists in using a centred difference operator in space while time marching with a Runge-Kutta scheme. The method is implemented on the interval $I=[0,1 / 2]$, with boundary conditions $\psi_{\nu}(0, t)=0$ and $\psi_{\nu}(1 / 2, t)=0$. The condition that $\psi_{v}(1 / 2, t)=0$ is consistent with the value of the dispersionless solution and as such is consistent for small enough values of $\epsilon$. We can use two different initial conditions:

$$
\begin{aligned}
& \psi(x, 0)=4 x^{3}-\frac{x}{t_{*}} \\
& \psi_{\nu}\left(x, t_{*}\right)=\frac{x}{t_{*}}-4 v x \sum_{n=1}^{\infty} \frac{1}{x^{2}-a_{n}^{2}\left(t_{*}, v\right)} .
\end{aligned}
$$

If the second condition is used, then the pole positions at $t=t_{*}$ are specified by the asymptotic estimate presented in property 4.1. This estimate is used for all values of $\mu_{n}$ 

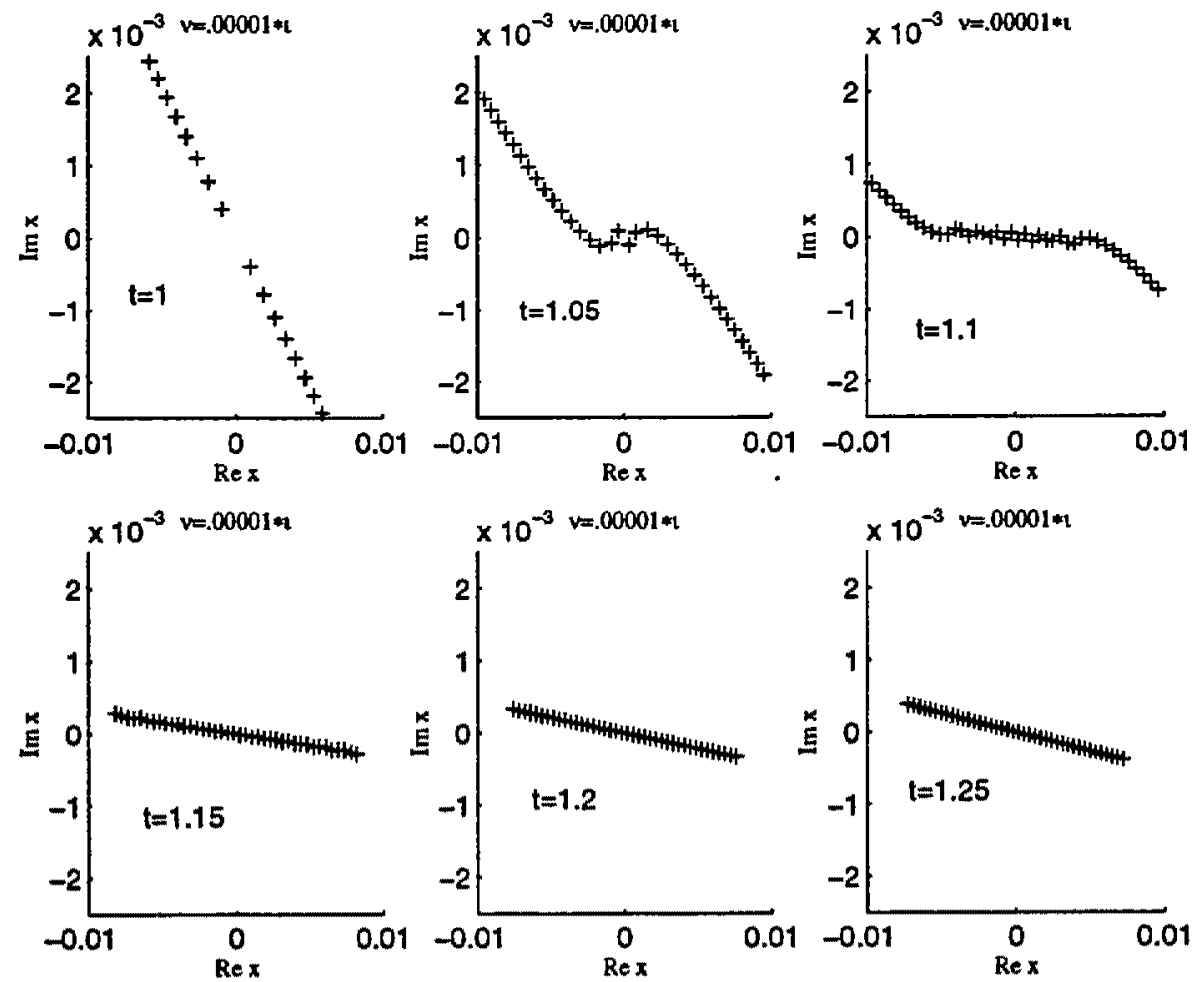

Figure 16. Positions of the first 20 poles from the pole dynamics for $v=10^{-5} \mathrm{i}$ and $N=10^{5}$ at $t=1,1.05,1.1,1.15,1.2,1.25$.

for $10 \leqslant n \leqslant N$ :

$$
\begin{aligned}
& a_{n}\left(t_{*}, \nu\right)=\mathrm{e}^{\mathrm{i} 3 \theta / 4} \mathrm{i} 4 t_{*}\left(2 \epsilon \mu_{n}\right)^{3 / 4}, \\
& \mu_{n}=G\left(\mu_{n}^{(0)}\right), \quad \mu_{n}^{(0)}=\frac{2 \pi}{3 \sqrt{3}}(n-1 / 3), \quad n \geqslant 10, \\
& G(\mu)=\mu+\frac{7}{432 \mu}\left(1-\frac{1}{6 \mu}\left(1+\frac{7}{72 \mu}\left(1-\frac{5}{12 \mu}\left(1+\frac{53143}{18900 \mu}\right)\right)\right)\right) .
\end{aligned}
$$

For $1 \leqslant n \leqslant 9$, we use the numerical values found in [28, table 3$]$, under the column headed Numerical roots:

$$
\begin{array}{lll}
\mu_{1}=0.8221037147 & \mu_{2}=2.0226889660 & \mu_{3}=3.2292915284 \\
\mu_{4}=4.4372464748 & \mu_{5}=5.6457167459 & \mu_{6}=6.8544374340 \\
\mu_{7}=8.0632985369 & \mu_{8}=9.2722462225 & \mu_{9}=10.4812510479
\end{array}
$$

Let

$$
\begin{aligned}
& \psi_{j}=\psi(j * \Delta x, t), \quad E \psi_{j}=\psi_{j+1}, \quad E^{p} \psi_{j}=\psi_{j+p}, \\
& D_{+}=\left(E-E^{0}\right) / \Delta x, \quad D_{-}=\left(E^{0}-E^{-1}\right) / \Delta x, \quad D_{0}=\left(D_{+}+D_{-}\right) / 2 .
\end{aligned}
$$

One then solves the system of $J-1$ equations using a Runge-Kutta 4-5 scheme:

$$
\frac{\mathrm{d} \psi_{j}}{\mathrm{~d} t}=-D_{0}\left(\psi_{j}^{2} / 2\right)+v D_{+} D_{-} \psi_{j}, \quad j=1, \cdots, J-1,
$$

where $J * \Delta x=1 / 2, \psi_{j=0}=0$ and $\psi_{J}=0$. 

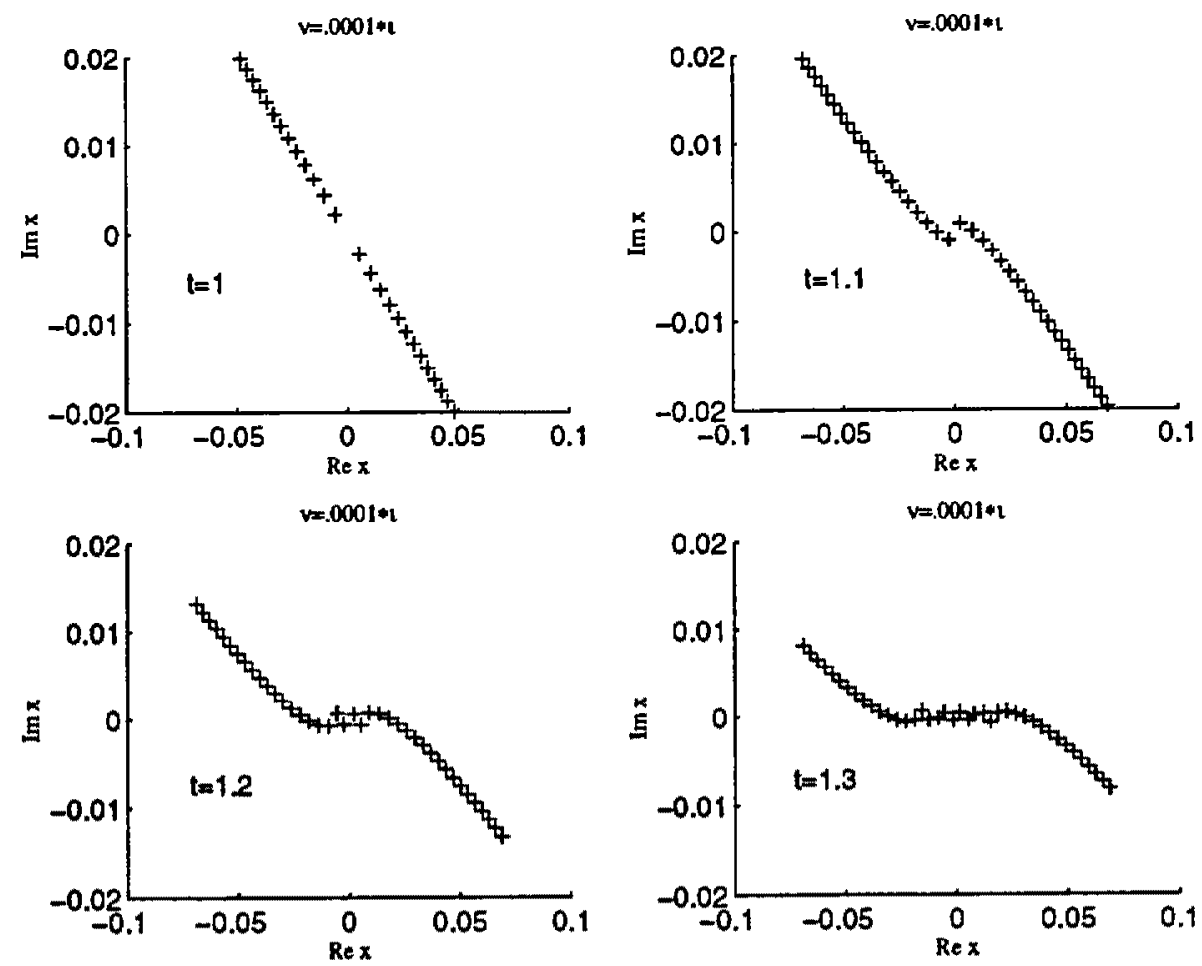

Figure 17. Positions of the first 20 poles from the pole dynamics for $v=10^{-4} \mathrm{i}$ and $N=10^{5}$ at $t=1,1.1,1.2,1.3$.

\subsection{Numerical pole dynamics}

We now investigate the motion of the simple poles of $\psi_{v}(x, t)$ for various values of $v \in \mathbb{C}^{+}$. The procedure consists in truncating the Calogero dynamical system and by starting with initial data for the poles at $t=t_{*}$ : the complex poles of $\psi_{v}(x, t)$ are located at $x= \pm a_{n}(t, v)= \pm \sqrt{\nu \kappa_{n}(t, v)}$, where the variables $\kappa_{n}$ satisfy the system

$$
\forall n \in \mathbb{N}^{*}, \quad\left\{\begin{array}{l}
\frac{1}{2} \frac{\mathrm{d} \kappa_{n}}{\mathrm{~d} t}=\frac{\kappa_{n}}{t}-1-4 \kappa_{n} \sum_{\substack{l=1 \\
l \neq n}}^{\infty} \frac{1}{\kappa_{n}-\kappa_{l}} \\
\kappa_{n}\left(t_{*}, v\right)=a_{n}^{2}\left(t_{*}, v\right) / \nu .
\end{array}\right.
$$

$a_{n}\left(t_{*}, v\right)$ is computed as is described in the previous section. The value of $a_{n}(t, v)$ is recovered using the relation $a_{n}(t, v)=\sqrt{v \kappa_{n}(t, v)}$. Starting from $t=t_{*}$, we compute and plot the evolution of the first four poles $a_{n}(t, v), n=-4, \cdots, 4$ for different values of $v$. We use $N$ poles in the computations, i.e. $a_{1}$ to $a_{N}$ where $N \times 10^{-4}$ is $1,2.5,5,10$. That is, we consider the truncated system

$$
\forall n=1, \cdots, N, \quad\left\{\begin{array}{l}
\frac{\dot{\kappa}_{n}}{2}=\frac{\kappa_{n}}{t}-1-4 \kappa_{n} \sum_{\substack{l=1 \\
l \neq n}}^{N} \frac{1}{\kappa_{n}-\kappa_{l}} \\
\kappa_{n}\left(t_{*}, v\right)=\left(4 t_{*}\right)^{2}\left(2 \mu_{n}\right)^{3 / 2} \sqrt{\epsilon} \mathrm{e}^{\mathrm{i} \theta / 2}
\end{array}\right.
$$

where the integer $N$ is appropriately chosen. In order to accelerate the computation of the slowly converging pole expansions

$$
\sum_{\substack{l=1 \\ l \neq n}}^{N} \frac{1}{\kappa_{l}-\kappa_{n}}, \quad \forall n=1, \cdots, N
$$





Figure 18. Positions of the first 20 poles from the pole dynamics for $v=10^{-3} \mathrm{i}$ and $N=10^{5}$ at $t=1,2,3,4$.

we use the Multipole algorithm developed by Greengard and Rokhlin [20] and implemented by Greengard, which reduces the number of operations from $\mathrm{O}\left(N^{2}\right)$ to $\mathrm{O}(N \log N)$. A fourth- and fifth-order Runge-Kutta-Fehlberg scheme with automatic step-size control is used. Since the initial data are specified at $t=t_{*}=1$, we can solve the system forwards and backwards in time starting from $t=1$. The typical tolerance in the computation is $10^{-8}<\left|\left(x_{4}-x_{5}\right) / x_{5}\right|<10^{-4}$ where $x_{4}$ and $x_{5}$ are the fourth- and fifth-order estimates of $\kappa_{1}(t, v)$, respectively. Once the tolerance criterion is met, we recover the pole location via the relation $a_{n}(t, v)=\sqrt{v \kappa_{n}(t, v)}$. The difference between the complex $v$ case and the real $v$ case is that the variables are all real for $v$ real, and thus system (7.4) is a system of real ODEs whereas, for $v \in \mathbb{C}^{+}$, system (7.4) is a genuinely complex ODE system. The justification of the numerics is the most difficult aspect of this simulation because one must justify the convergence of the method both as the number of poles increases and as the time step is refined. The time-step control is automatically determined by the relative tolerance (RT) test on the fourth- and fifth-order approximations of the first ordered pole (the one closest to the origin). (Note that RT is also called LRT for local relative tolerance in the figure captions.) Thus one cannot fix the time stepping; rather one can have a subtle control on it by reducing this tolerance. Typically, we fix the number of poles to 50000 and vary the tolerance on the successive intervals $10^{-10}<\mathrm{RT}<10^{-6}, 10^{-8}<\mathrm{RT}<10^{-4}, 10^{-6}<\mathrm{RT}<10^{-2}$. Then we fix the tolerance at the highest reasonable level $10^{-8}<\mathrm{RT}<10^{-4}$, and vary the number of poles where $N \times 10^{-4}$ varies from 1,2.5,5,10. Another test of accuracy is performed on exactly solvable two-pair pole dynamics [29 (I, section 5)] and can easily be adapted to this case. A discussion of the convergence of the pole dynamics method for the case 


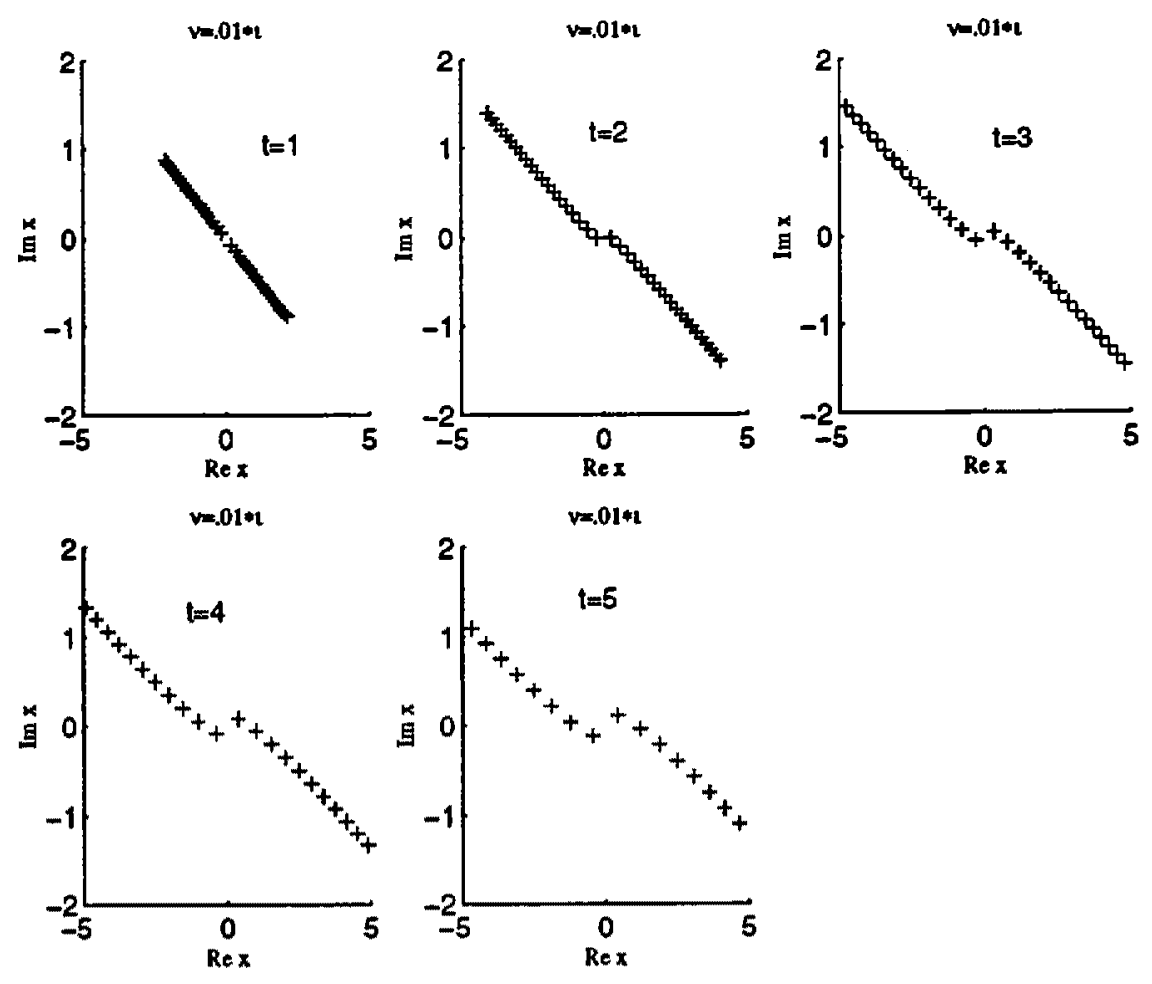

Figure 19. Positions of the first 20 poles from the pole dynamics for $v=10^{-2} \mathrm{i}$ and $N=10^{5}$ at $t=1,2,3,4,5$.

$v \in \mathbb{R}$ can be found in $[29, \mathrm{I}]$. The convergence of the (truncated) pole dynamics to the true solution of the (infinite) Calogero dynamical system as the number $N$ of poles increases and as the time stepping of the Runge-Kutta scheme is decreased improves as the argument $\theta$ of $v$ increases to $\pi / 2$ and worsens as the magnitude $\epsilon$ of $v$ decreases. The convergence improves with increasing $\theta$ because the position of the poles at $t_{*}$ gets closer to the real axis. The closer the poles are to the real axis, the better the convergence in the tails of the solution becomes. Indeed, the most difficult case (computationally) occurs when $\arg v=0$, as discussed in $[29, \mathrm{I}]$.

When $v>0$, the behaviour of the pole $\beta_{1}(t, v)$ displayed in [29, I] describes the evolution of the width of the analyticity strip of the viscous solution.

When $v \in \mathrm{i} \mathbb{R}$, the behaviour of the poles is studied as $\epsilon=|v|$ decreases to 0 . One can observe a structuring of the pole behaviour into a spiralling motion at the end of which they end close to the real axis for $t \approx t_{*}, t>t_{*}$. It is the presence of these poles close to the real axis which gives rise to rapid oscillations which are observed in both the pole expansion reconstruction and the stationary phase approximation. We describe many cases for $\epsilon$ ranging from $\epsilon=10^{-2}$ to $\epsilon=10^{-5}$ due to the drastic difference in the behaviour of the poles displayed in figures 2-7. In many of these figures, we do not display the full height of the oscillations in order to compare the pole dynamics with the stationary phase approximation. The agreement between the two methods is remarkable (figures 8-10). The few discrepancies which can be observed in these figures occur in the amplitude of the peaks of certain oscillations. They are due to the extreme sensitivity of the pole reconstruction to 




Figure 20. $\Im a_{j}(t, v)$ versus $\Re a_{j}(t, v)$. Time evolution in $\mathbb{C}$ of $a_{j}(t, v), j=-4, \cdots, 4$ for $v=10^{-3} \mathrm{e}^{\mathrm{i} \pi / 4}$ and $N=10^{5}$. $t_{\text {initial }}=t_{*}=1$ and $t_{\text {final }}=4 . n_{\text {steps }}=80$. Time-stepping tolerance: $10^{-8}<\mathrm{LRT}<10^{-4}$.

the pole positions. The accuracy of the match between the stationary phase approximation and the pole dynamics also serves as a justification for the pole dynamics.

Finally, only the case $\epsilon=10^{-3}$ is treated for $\arg v=\pi / 4$ to illustrate the behaviour of a mixed dissipative-dispersive system (see (3.3)). In this case ( $\arg v=\pi / 4$ ), the diversity in the behaviour of the poles in figure 20 is much less rich than that observed for $\arg v=\pi / 2$. Moreover, the number of oscillations is fixed to one and, as such, is less interesting to observe. However, this case is included to provide a comparison with the (full) finitedifference scheme (method of lines) displayed in figure 21.

\subsection{Numerical branch-cut dynamics}

Finally we present the results of numerical computations for the branch-cut dynamics equation. Rather than solving (6.8) directly, we move points $X(t)$ on the branch cut through the equation

$$
\dot{X}(t)=\frac{1}{2}\left(\psi_{+}+\psi_{-}\right)(X(t), t),
$$

in which $\psi_{+}$and $\psi_{-}$are the limits from the right and left, respectively, of the corresponding solution of the inviscid Burgers equation. For the initial data $\psi(x, 0)=4 x^{3}-x / t_{*}$, the positions of the poles are prescribed at $t=t_{*}$ to be the pole positions for the Burgers equation with viscosity $v=\epsilon \mathrm{e}^{\mathrm{i} \theta}$, as described in section 4 and [28]. In particular they lie on the line $\arg z=3 \theta / 4$. Their location for $t>t_{*}$ is found by solving the ODE (7.5). Moreover the solution values

$$
\psi(x, t)=\psi_{0}\left(x_{0}\right)=4 x_{0}^{3}-x_{0} / t_{*}
$$



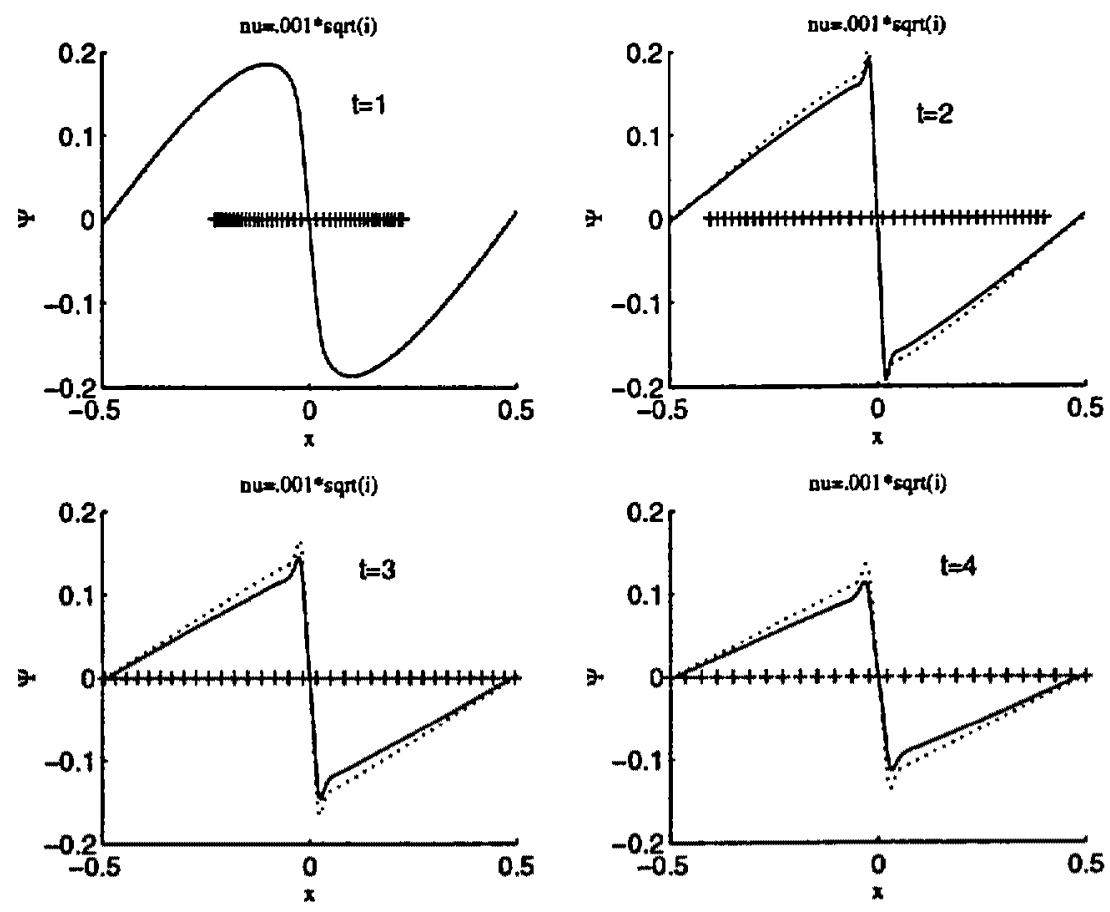

Figure 21. $\Re \psi_{v}(x, t)$ versus $x$. Comparison of pole dynamics $(-)$ and finite-difference approximation $(\cdots \cdots)$ for $v=10^{-3} \mathrm{e}^{\mathrm{i} \pi / 4}$ and $N=10^{5}$ at $t=1,2,3,4$. Projection of pole locations on the real axis $(+)$.

are found through the inversion of the cubic equation [29, section 6]

$$
x=x_{0}+t \psi_{0}\left(x_{0}\right)=4 t x_{0}^{3}+x_{0}\left(t_{*}-t\right) / t_{*} .
$$

At a complex point $x$ on the branch cut we have

$$
\psi_{+}(x, t)=\psi_{0}\left(x_{+}(x, t), t\right), \quad \psi_{-}(x, t)=\psi_{0}\left(x_{-}(x, t), t\right),
$$

in which $x_{+}$and $x_{-}$are the limiting values of $x_{0}$ from the right and left at the point $x$. For large positive or negative values of $x$ on the real line, the cubic equation (7.7) has a single real value $x_{0}=x_{0}(x, t)$. The value $x_{+}(x, t)$ is the analytic continuation of this real value of $x_{0}(x, t)$ from the positive real axis; the value $x_{-}(x, t)$ is the analytic continuation of $x_{0}(x, t)$ from the negative real axis.

Results of numerical solution of the branch-cut dynamics equation in the form (7.5) corresponding to initial data (7.6) are presented in figures $11-14$ for $v=10^{-5} \mathrm{i}, 10^{-4} \mathrm{i}, 10^{-3} \mathrm{i}$ and $10^{-3} \sqrt{\mathrm{i}}$. As described in section 6.1, the equation for the branch cut depends only on $\theta=\arg v$; the value of $\epsilon$ is only used to determine the positions of the poles at $t=t_{*}$ * corresponding to that value of $\nu$. In each of these figures, as well as in similar computations for other values of $v$, we see that the branch cut is a line of angle $3 \theta / 4$ at $t=t_{*}$ and that, as $t$ increases, the branch cut again approaches a line but with a small angle. For $\theta=\pi / 2$ (i.e. $v=\mathrm{i} \epsilon$ ) the branch cut moves onto the real line as $t$ increases.

Next we compare the pole positions computed by the branch-cut dynamics method with those obtained from the Calogero equations. The Calogero system is exact, except for discretization in $t$ and a cut-off in the number $N$ of poles. In the case $v=10^{-3} \sqrt{\mathrm{i}}$, there is excellent agreement between the results from the branch-cut dynamics (figure 14) and 
those from the Calogero system (figure 15). The cases with $v=\mathrm{i} \epsilon$ are more interesting, since there are oscillations in the corresponding Burgers solution. The branch-cut dynamics results of figures 11-13 are in excellent agreement with the Calogero results of figures 1618 for poles that are outside the caustic points of the inviscid Burgers solution. Within the caustic region, the poles from the branch-cut dynamics lie on the real axis, while those from Calogero lie slightly off. On the other hand, the real parts of the pole positions from the two methods are in good agreement.

This shows that the branch-cut dynamics do a very good job of describing the pole dynamics for Burgers equation with complex viscosity, except within the caustic region for imaginary viscosity.

\section{Acknowledgments}

The research of R Caflisch and D Senouf was partially supported by NSF grant No DMS9306720. The research of N Ercolani was partially supported by grant No DMS-9302013. The first author gratefully acknowledges Professor L Greengard for the expeditious use of his Multipole code which greatly enhanced the study of the numerical pole dynamics, and Dr Anders Peterson for many valuable conversations concerning the method of lines.

\section{Appendix. Generalization of the initial data to $\psi_{0}(x)=2 n x^{2 n-1}-x / t_{*}$}

Using a result in [29, I] concerning the asymptotic behaviour of the $k$ th zero $\mu_{k, n}$ of $\mathcal{F}_{n}(\mu)=\int_{-\infty}^{\infty} \mathrm{e}^{\mu\left(2 n i z-z^{2 n}\right)} \mathrm{d} z$, we can prove the following.

Property A.1. Let $n \in \mathbb{N}, n \geqslant 2$, and let $v=\epsilon \mathrm{e}^{\mathrm{i} \theta} \in \mathbb{C}^{+}=\{\epsilon>0,|\theta| \leqslant \pi / 2\}$. The kth ordered pole of the solution at $t=t_{*}$ arising from the initial data $\psi_{0}(x)=2 n x^{2 n-1}-x / t_{*}$ is located at

$$
a_{k, n}\left(t_{*}, v=\epsilon \mathrm{e}^{\mathrm{i} \theta}\right)=\mathrm{e}^{\mathrm{i} \frac{2 n-1}{2 n} \theta} \mathrm{i} 2 n t_{*}\left(2 \epsilon \mu_{k, n}\right)^{\frac{2 n-1}{2 n}},
$$

where the coefficients $\mu_{k, n}$ are asymptotically given by

$$
\mu_{k, n}=\frac{\pi}{4 n-2} \sec \left(\frac{\pi}{4 n-2}\right)\left(\frac{n-1}{2 n-1}+1+2 k\right)+\mathrm{O}\left(\frac{1}{k}\right) \text { as } k \rightarrow+\infty \text {. }
$$

\section{References}

[1] Abramowitz M and Stegun I A 1964 Handbook of Mathematical Functions (New York: Dover)

[2] Ahlfors L V 1979 Complex Analysis 3rd edn (New York: McGraw-Hill)

[3] Bessis D and Fournier J D 1990 Complex singularities and the Riemann surface for the Burgers equation Research Reports in Physics-Nonlinear Physics (Berlin: Springer) pp 252-7

[4] Bessis D and Fournier J D 1984 Pole condensation and the Riemann surface associated with a shock in Burgers equation J. Phys. Lett. 45 L833-41

[5] Boas R P 1954 Entire Functions (New York: Academic)

[6] Burgers J M 1948 A mathematical model illustrating the theory of turbulence Adv. Appl. Mech. 1 171-99

[7] Burgers J M 1974 The Nonlinear Diffusion Equation (Dordrecht: Reidel)

[8] Caflisch R E, Ercolani N, Hou T Y and Landis Y 1993 Multi-valued solutions and branch point singularities for nonlinear hyperbolic or elliptic systems Commun. Pure Appl. Math. 46 453-99

[9] Caflisch R E and Lowengrub J S 1989 Convergence of the vortex method for vortex sheets SIAM J. Numer. Anal. 26 1060-80

[10] Calogero F 1978 Motion of poles and zeros of special solutions of nonlinear and linear partial differential equations and related 'solvable' many body problems Nuovo Cimento B 43 177-241 
[11] Choodnovsky D V and Choodnovsky G V 1977 Pole expansions of nonlinear partial differential equations Nuovo Cimento B 40 339-53

[12] Cole J D 1951 On a quasi-linear parabolic equation occuring in aerodynamics Quart. Appl. Math. 9 225-36

[13] Copson E T 1965 Asymptotic Expansions (Cambridge: Cambridge University Press)

[14] Copson E T 1957 An Introduction to the Theory of Functions of a Complex Variable (Oxford: Oxford University Press)

[15] Dobrokhotov S Y, Maslov V P and Tsvetkov V B 1992 Problem of the reversal of a wave for the model equation $v_{t}+v v_{x}-\frac{\mathrm{i} h}{2} v_{x x}=0$ Mate. Zametki 51 143-7

[16] Ercolani N M, Gabitov I R, Levermore C D and Serre D (ed) 1994 Singular Limits of Dispersive Waves (NATO ASI Ser. B 320)

[17] Ercolani N M, Levermore C D and Zhang T 1994 The behaviour of the Weyl function in the zero-dispersion KdV limit MSRI Preprint 045-94; 1996 Commun. Math. Phys. at press

[18] Fournier J D and Frisch U 1983 L'équation de Burgers deterministe et statistique J. Mec. Th. Appl. 2 699-750

[19] Frisch U and Morf R 1981 Intermittency in nonlinear dynamics and singularitites at complex times Phys. Rev. A 23 2673-705

[20] Greengard L and Rokhlin V 1987 A fast algorithm for particle simulations J. Comput. Phys. 73 325-48

[21] Hopf E 1950 The partial differential equation $u_{t}+u u_{x}=\mu u_{x x}$ Commun. Pure Appl. Math. 3 201-30

[22] Jin S, Levermore C D and McLaughlin D W 1994 The behaviour of solutions of the NLS equation in the semiclassical limit Singular Limits of Dispersive Waves ed N M Ercolani et al (New York: Plenum) pp 235-55

[23] Kaminski D 1989 Asymptotic expansion of the Pearcey integral near the caustic SIAM J. Math. Anal. 20 987-1005

[24] Lax P D and Levermore C D 1983 The small dispersion limit of the Korteweg de Vries equation, I, II, III Commun. Pure Appl. Math. 36 253-90, 571-93, 809-29

[25] Levermore C D and Liu J G 1994 Oscillations arising in numerical experiments Singular Limits of Dispersive Waves ed N M Ercolani et al (New York: Plenum) 329-46

[26] Paris R B 1991 The asymptotic behaviour of Pearcey's integral for complex variables Proc. R. Soc. Ser. A 432 391-426

[27] Pólya G 1927 Über trigonometrische integrale mit nur reellen nullstellen J. Reine Angewandte Mathe. 158 6-18

[28] Senouf D 1996 Asymptotic and numerical approximations of the zeros of Fourier integrals SIAM J. Math. Anal. 27 1102-28

[29] Senouf D 1997 Dynamics and condensation of complex singularities for Burgers equation. I \& II SIAM J. Math. Anal. to appear

[30] Sulem C, Sulem P L and Frisch H 1983 Tracing complex singularities with spectral methods J. Comput. Phys. 50 138-61

[31] Thual O, Frisch U and Hénon M 1985 Application of pole decomposition to an equation governing the dynamics of wrinkled flame fronts $J$. Physique 46 1485-94

[32] Ursell F 1972 Integrals with a large parameter. Several nearly coincident saddle points Proc. Cambridge Phil. Soc. 72 49-65

[33] Whitham G B 1974 Linear and Nonlinear Waves (New York: Wiley-Interscience) 